\title{
Insider Trading Restrictions and Analysts' Incentives to Follow Firms
}

\author{
ROBERT M. BUSHMAN, JOSEPH D. PIOTROSKI, and ABBIE J. SMITH*
}

\begin{abstract}
Motivated by extant finance theory predicting that insider trading crowds out private information acquisition by outsiders, we use data for 100 countries for the years 19872000 to study whether analyst following in a country increases following restriction of insider trading activities. We document that analyst following increases after initial enforcement of insider trading laws. This increase is concentrated in emerging market countries, but is smaller if the country has previously liberalized its capital market. We also find that analyst following responds less intensely to initial enforcement when a country has a preexisting portfolio of strong investor protections.
\end{abstract}

THE AVAILABILITY OF INFORMATION IS A KEY determinant of the efficiency of resource allocation decisions in economies and their securities markets. There is, however, considerable cross-country variation in the quality and quantity of corporate reporting, information intermediation, and information dissemination structures (e.g., Bushman, Piotroski, and Smith (2004)). Hence an important research task is to understand why information infrastructures vary and what factors determine their evolution over time.

In this paper, we focus on one element of information infrastructure, sellside analysts. We exploit cross-country and intertemporal variation in analyst following for 100 countries for the years 1987-2000 to test the hypothesis that analyst following increases upon the restriction of insider trading. Our analysis specifically tests whether analyst following increases after adoption of insider trading legislation and/or the initial act of enforcement of these laws.

This research builds directly on three prior strands of research. The first is theoretical research predicting that insider trading crowds out private information acquisition by outside investors (e.g., Fishman and Hagerty (1992) and Khanna, Slezak, and Bradley (1994)). In these papers, insiders with free access to information about a firm's payoff reduce trading profits available to

\footnotetext{
* Bushman is at the University of North Carolina Kenan-Flagler Business School, and Piotroski and Smith are at the University of Chicago Graduate School of Business. We thank Jeff Abarbanell, Mark Lang, Darius Miller, Jake Thomas, Jerry Zimmerman, an anonymous referee, and workshop participants at University of Chicago, University of Colorado, Harvard Business School, University of Illinois (Chicago), University of Rochester, Stanford University, UCLA, and Yale University for helpful discussions, comments, and suggestions. We also appreciate the research assistance of Sara Eriksen and the financial support of Harvard Business School, Kenan-Flagler Business School, the William Ladany Faculty Research Fund, and the Institute of Professional Accounting of the GSB, University of Chicago.
} 
outside traders from costly information acquisition. As a result, introducing insiders leads to an equilibrium in which fewer traders acquire private information signals. Consistent with this prediction, Bhattacharya et al. (2000) examine shares trading on the Bolsa Mexicana de Valores and provide evidence that these share prices do not react to company news. Their evidence suggests that unrestricted insider trading causes prices to fully incorporate firm-specific information before its public release, thereby limiting the gains to private information acquisition activities. Related research (e.g., Brudney (1979), Ausubel (1990), and Leland (1992)), although it does not specifically address information intermediaries, also argues that insider trading generally reduces participation in equity markets. To the extent that sell-side analysts map into the construct of costly information collectors and processors competing for a share of informed trading profits or proxy for equity market participation in general, these theories imply that analyst following will increase after restriction of insider trading activities. Because insider trading research focusing on U.S. markets offers limited opportunities to examine significant changes in insider trading regimes, we employ cross-country panel data on the initial enactment and enforcement of insider trading laws. This country-level research design provides us with a novel opportunity to examine the theory that insider trading crowds out information intermediation.

The second research strand examines responses to changes in insider trading laws. Closely related to our study, the work of Bhattacharya and Daouk (2002) documents that initial enforcement (but not enactment) of insider trading laws is associated with a significant decrease in country-level cost of equity capital. Given this important result, we take the logical next step of investigating potential channels through which insider trading laws act to produce this reduction in the cost of capital. While insider trading restrictions could impact the cost of capital through a number of channels, information intermediation by analysts represents one potentially important channel. ${ }^{1}$ Increased analyst activity can reduce the incidence of neglected stocks in portfolio formation (Merton (1987)), estimation risk (Barry and Brown (1985)), and/or undiversifiable risk born by uninformed investors due to informed investors being better able to shift portfolio weights to incorporate new information (Easley, Hvidkjaer, and O'Hara (2002), Easley and O'Hara (2004)). In addition, research has documented smaller bid-ask spreads and greater liquidity for highly followed firms (Brennan and Subrahmanyam (1995), Roulstone (2003)).

Finally, our investigation of economy-wide changes in analyst following upon shifts in insider trading legislation and enforcement contributes to the growing

\footnotetext{
${ }^{1}$ For example, insider trading restriction may directly reduce the adverse selection component of the bid/ask spread (e.g., Kyle (1985)). Beny (1999) finds that tougher insider trading laws are negatively associated with ownership concentration consistent with reduction in benefits of control. Maug (1999) argues that with unregulated insider trading, managers can bribe large shareholders to refrain from monitoring their actions by warning them early about negative developments. Thus, a direct governance response could contribute to the economy-wide impacts documented. Bushman et al. (2004) find cross-country evidence of a positive correlation between enforcement of insider trading restrictions and corporate disclosure, institutional investing, and media penetration.
} 
literature that examines relations between legal regimes and financial market characteristics (e.g., Beck, Demirgüç-Kunt, and Levine (2001, 2003), DemirgüçKunt and Maksimovic (1998), La Porta et al. (1997, 1998), and Lombardo and Pagano (1999)).

We document that both the intensity of analyst coverage (the average number of analysts covering followed firms within a country) and the breadth of coverage (the proportion of domestic listed firms followed by analysts) increase after initial enforcement of insider trading laws. ${ }^{2}$ We further find that this increase is concentrated in emerging market countries. These results are robust to inclusion of an array of control variables and rigorous specification checks designed to rule out the possibility that our results simply capture a general upward trend in actual analyst coverage or in reported analyst coverage by I/B/E/S International. ${ }^{3}$ In particular, we trend-adjust analyst coverage for each country-year observation using countries that enforced insider trading prior to our sample period as coverage benchmarks, we utilize bootstrap procedures to test the significance of our results, and we reestimate our main regressions using only the most recent time periods (1992-2000).

It is of course possible that both analyst following and insider trading restrictions are driven by the same correlated omitted variable. We show that our results are robust to several alternative explanations. First, we control for the date of countries' liberalization of their equity market (if they did liberalize). Financial liberalization gives foreign investors the opportunity to invest in domestic markets and domestic investors the right to transact in foreign securities. This is a fundamental national policy decision often enacted simultaneously with other macro-economic policies. ${ }^{4}$ Our results are robust to the inclusion of a liberalization variable. Moreover, we find that the increase in analyst following after insider trading enforcement is smaller in countries that have already liberalized.

Second, we examine measures of actual financial flows, as liberalization may not impact all countries similarly. We include the flow of foreign direct investment into a country to control for the possibility that inflows of foreign capital both draw more analysts and simultaneously pressure governments to crack down on insider trading to please foreign capital providers. Following Rajan and Zingales (2002), we also consider a measure of the openness of countries' product

\footnotetext{
${ }^{2}$ We find modest evidence of changes in analyst following around enactment of insider trading laws. However, the results for enforcement are more robust across the entire range of specifications examined. This is consistent with Bhattacharya and Daouk (2002), who find that the equity cost of capital decreases after enforcement but not after adoption. Estrada and Pena (2001) find no systematic reaction to the adoption or tightening of insider trading rules in 10 European countries. They do not examine enforcement events although they recognize that enforcement is likely to be a significant factor.

${ }^{3}$ We acknowledge the possibility that an increase in reported analyst coverage by I/B/E/S is not necessarily equivalent to the initiation of new coverage by analysts. We discuss this issue and its implications for the interpretation of our results in Section II.

${ }^{4}$ See Bekaert, Harvey, and Lundblad (2001) for a detailed discussion. They find that the economic growth of a country significantly increases after liberalization. They also find that economic growth increases after insider trading enforcement, after controlling for financial liberalization.
} 
markets (imports plus exports scaled by GDP). As product flows increase, the incentives for incumbents to oppose financial development (like insider trading laws) diminish as they are willing to trade financial development concessions to gain benefits from increased market possibilities. Our results are robust to these controls for the level of foreign direct investment and trade openness.

Finally, we investigate whether the analyst response to changes in insider trading regimes is stronger or weaker when a country already has strong investor protections. We use a country's legal origin to proxy for the level of investor protection (e.g., La Porta et al. (1998, 1999)), whereby investor protections are presumed to be stronger in countries with a common law origin relative to countries with either civil law or socialist legal origins. We document that analyst following increases more intensely upon enforcement of insider trading restrictions in countries with civil law or socialist legal origins relative to countries with a common law origin.

The rest of the paper is organized as follows. Section I discusses additional pertinent background literature and research design considerations. Section II describes our measures of analyst activity and provides descriptive statistics for regression model variables. Section III presents and discusses the main empirical results of the paper, and Section IV concludes.

\section{Background and Research Design Considerations}

Our main hypothesis is that analyst following increases when insider trading is restricted. In Section I.A, we fully develop this hypothesis and discuss related research. In Section I.B, we discuss limitations of our data on insider trading activities and define our insider trading enactment and enforcement variables.

\section{A. Do Analysts Map into the Construct of Privately Informed Traders?}

A large body of research uses analyst coverage as a proxy for the existence of informed market participants, underlying investor interest, or private information acquisition activities. In general, this research documents that greater analyst following is associated with an improvement in the flow of information into prices. This relation is reflected by a smaller price response to earnings for firms with analyst coverage (Lobo and Mahmoud (1989)), faster incorporation of common information into price (Brennan, Jegadeesh, and Swaminathan (1993)), more timely prices with respect to future earnings (Ayers and Freeman (2003)), and smaller anomaly related returns (e.g., momentum (Hong, Lim, and Stein (2000)) and value investing strategy (Piotroski (2000)). Thus, empirical evidence supports the view that analysts provide valuable information to market participants.

However, it is possible that financial analysts do not map into the theoretical construct of investors who gather and trade on private information. For example, Easley, O'Hara, and Paperman (1998) argue that analyst following is not a good proxy for information-based trading, and conjecture that the fundamental role of analysts may be to showcase stocks to uninformed investors. 
Brennan and Subramanyam (1995) and Roulstone (2003) document smaller bid-ask spreads and greater liquidity for firms with higher analyst following, which does not appear consistent with an adverse selection component in the bid-ask spreads driven by analysts possessing significant private information. Also, insider traders and financial analysts may not directly compete for the same information set. For example, Piotroski and Roulstone (2004) document that insider trading increases the relative amount of firm-specific information being impounded into prices, while analyst forecasting activity leads to a greater relative amount of industry and market-level information influencing prices. Thus, if analysts are showcasing devices rather than private information traders, or if analysts focus on disseminating macro-level information while inside traders focus on firm-specific information, a substitute relation between insider trading activities and analyst following may, but need not, exist.

Another possibility is that even if a substitute relation between insider trading activities and private information collection/analyst following does exist, such a relation need not exist between the adoption/enforcement of insider trading laws and private information collection incentives if the adoption and enforcement of insider trading laws does not significantly reduce insider trading. ${ }^{5}$ Consistent with this possibility, Bris (2000) presents evidence that after initial enforcement of insider trading laws, insiders appropriate a larger portion of the total gains in takeover transactions than they did before enforcement. ${ }^{6}$ However, if enforcement of insider trading laws has no impact on the extent of insider trading or if it increases such trading, it is difficult to understand the channels through which such enforcement would change liquidity in the first place.

In the end, the relation between adoption and enforcement of insider trading laws and financial analysts is an empirical question addressed in this paper.

\section{B. Limitations of Insider Trading Data and Variable Definitions}

The insider trading data we use is drawn from Bhattacharya and Daouk (2002) and consist of the date on which a country adopted insider trading legislation and the date of the initial prosecution under these laws, whether successful or not. They document that at the end of 1998, of 103 countries with existing stock markets, 87 have insider trading laws, while only 38 have enforced these

\footnotetext{
${ }^{5}$ Even in the United States with its strict insider trading laws and aggressive enforcement mechanism, all illegal insider trading is not driven away (see, e.g., Meulbroek (1992) and Keown and Pinkerton (1981)).

${ }^{6}$ Bris (2000) argues that strict enforcement of insider trading laws could create monopoly profits for traders who can skirt detection, increasing incentives for insider trading. He also argues that the documented increase in market liquidity after enforcement of insider trading laws could increase incentives for insider trading, given results in Kyle (1985) and others that an increase in liquidity trading increases the trading profits of an informed trader with private information of a given quality.
} 
laws. Before 1990, the numbers were 34 and 9 . We include their data set in Appendix B. ${ }^{7}$

A potential limitation of these data is that the adoption and initial enforcement of insider trading restrictions may have a small effect on insider trading activities. Bhattacharya and Daouk (2002) indicate that they would prefer to use data on how consistent and intensive insider trading enforcement is in a country; however, they are forced to compromise due to difficulties in extracting these data from countries' regulators.

Specifically, the dates of adoption and enforcement of insider trading restrictions fail to capture substantial differences in the nature of the insider trading laws and related enforcement mechanisms. For example, Maug (1999) isolates important differences in existing insider trading regimes. One important aspect is whether insider trading prosecution is criminal or civil, and the resultant impact the prosecution mechanism has on the legal definition of insider trading. Maug demonstrates that these types of distinctions in insider trading laws can have significant implications for the ultimate impact of insider trading laws on economic welfare. ${ }^{8}$ Bris (2000) notes significant differences in insider trading laws across countries relative to who is considered an insider. Estrada and Pena (2001) also document significant differences in the precise details of insider trading laws across 10 European countries. The task of exploring the underlying details of insider trading laws and enforcement is beyond the scope of this paper.

Despite the apparent limitations of our insider trading data, recent evidence in Bhattacharya and Daouk (2002) and Bekaert, Harvey, and Lundblad (2001) suggests that the enforcement date data capture meaningful differences in insider trading regimes around the world. To the extent that these data are noisy measures of the actual curtailment of insider trading activities, we expect the power of our tests to be reduced. In an attempt to deal with this noisy date issue, we assess the statistical significance of observed relations using bootstrapping techniques.

Using the data from Bhattacharya and Daouk (2002), we define two sets of enactment and enforcement variables. The first set of indicator variables is set equal to 1 in a given country in the years during and after the initial enactment (Exist) or enforcement (Enforce) of insider trading laws, and 0 in all prior years. These indicator variables switch on in the year of the insider trading event, and stay on for the remaining sample years. We use these insider trading regime indicators to document shifts in the level of analyst activity over time. The second set of indicator variables are set equal to 1 in a given country in the year of enactment ( $\Delta$ Exist) or initial enforcement ( $\Delta E$ Enforce) of insider trading restrictions, and set equal to 0 in all other years. These indicator variables switch on only in the year of the insider trading event. We use these variables

\footnotetext{
${ }^{7}$ We dropped three countries, Palestine, Taiwan, and Yugoslavia, due to lack of data availability, and so limit ourselves to the 100 countries listed in Appendix B.

${ }^{8}$ Other theoretical papers focusing on insider trading enforcement are DeMarzo, Fishman, and Hagerty (1998) and Shin (1996), which derive optimal enforcement strategies for given insider trading restrictions.
} 
to measure the incremental changes in analyst activities around these insider trading events.

\section{Measures of Analyst Activity and Descriptive Statistics for Regression Model Variables}

Data on financial analysts come from I/B/E/S International (Appendix A lists all variables, definitions, and data sources). I/B/E/S reports analyst data for 54 countries in our sample. We construct two measures of analyst following using analyst forecast data for every country and every year over the period 1987-2000 for which data are available on I/B/E/S. To capture the intensity of analyst following, our first measure, denoted Analyst $_{i, t}$, is the mean number of analysts providing a forecast for each firm listed on I/B/E/S for a given country $i$, in calendar year $t$. To capture the breadth of analyst following, our second measure, Proport $_{i, t}$, is the proportion of firms in country $i$ covered by an analyst in calendar year $t$. This proportion is measured as Ibesfirm $_{i, t} /$ Listed $_{i, t}$, where Ibesfirm $_{i, t}$ is the number of firms in country $i$ that are included in $\mathrm{I} / \mathrm{B} / \mathrm{E} / \mathrm{S}$ in calendar year $t$, and Listed $_{i, t}$ is the number of domestic companies in country $i$ listed on the home country's stock exchange during calendar year $t .^{9}$

We make several assumptions in constructing these measures. First, if a country is not represented on $\mathrm{I} / \mathrm{B} / \mathrm{E} / \mathrm{S}$ in a given year but does have a stock exchange, we assume that there is no analyst following in that country in that year. Second, any firm not listed in $\mathrm{I} / \mathrm{B} / \mathrm{E} / \mathrm{S}$ in a given year is assumed to have no analyst following that year. By implication, we are assuming that a firm added to the I/B/E/S database had no analyst coverage in the years before its addition.

Discussions with First Call (formerly I/B/E/S) indicate that in fact many additions represent new coverage of the firm. However, additions to the database can also occur at the time analysts' firms (e.g., brokerages) choose to become contributors to I/B/E/S. New contributors may have existing analyst coverage of firms that was not reported by I/B/E/S prior to the contributor joining I/B/E/S. First Call indicates that these different types of coverage initiation cannot be reliably distinguished.

This raises two issues. First, it may be the case that noncontributing analysts are following firms in a given country preenforcement, and these analysts choose not to become I/B/E/S contributors after insider trading enforcement. To the extent that these analysts increase coverage after initial enforcement, or some analysts initiate coverage after initial enforcement but choose not to contribute to $\mathrm{I} / \mathrm{B} / \mathrm{E} / \mathrm{S}$, this will work against our finding a result, as we treat these events as no change in coverage when in fact there was an increase in coverage.

\footnotetext{
${ }^{9}$ Note that Ibesfirm includes domestic firms, subsidiaries of foreign multinationals, and crosslisted multinationals that are included on $\mathrm{I} / \mathrm{B} / \mathrm{E} / \mathrm{S}$, while the denominator represents only the number of domestic companies, introducing noise into the measure Proport.
} 
Second, if there is an increase in analyst coverage as reported by I/B/E/S following enforcement of insider trading laws, we cannot disentangle the extent to which this represents the initiation of new coverage or the decisions of analysts following firms preenforcement to become contributors to I/B/E/S postenforcement. Even though we cannot disentangle them, both decisions represent an escalation in equity market participation by analysts. That is, the enforcement of insider trading laws may create incentives for analysts to follow more firms, or to increase their prominence or prestige in the market by becoming a contributor to I/B/E/S, or for I/B/E/S to expend additional efforts to increase the number of contributors to the database. The fact that these decisions follow the restriction of insider trading is consistent with existing finance theory predicting an increase in equity market participation upon restriction of insider trading (e.g., Fishman and Hagerty (1992), Khanna et al. (1994), Brudney (1979), Ausubel (1990), and Leland (1992)).

Another important issue with using $\mathrm{I} / \mathrm{B} / \mathrm{E} / \mathrm{S}$ data in our research design is the effect of trends in the data. To convincingly isolate any increase in analyst following after insider trading restrictions, it is necessary to rule out the possibility that our results simply capture a general upward trend in actual analyst coverage or in reported analyst coverage by I/B/E/S International. In our analyses, we take special care to rule out this possibility. As discussed in more detail below, we include variables designed to capture macro-effects, including growth in GNP, number of firms listed on the domestic exchange, and annual indicator variables. In addition, we trend adjust our analyst coverage metrics for each country-year observation, utilize bootstrap procedures, and reestimate the primary models using only more recent time periods (1992-2000).

Table I, Panel A provides descriptive statistics for the analyst variables along with other country characteristics. It is evident that analyst following varies widely across the sample, as do the other country-level characteristics considered. There is no analyst following for over half of the sample observations, with a mean country-level following (Analyst) of 2.672 analysts per firm, a standard deviation of 3.676 analysts per firm, and a maximum average of over 17 analysts per firm. Similarly, the average proportion of firms with I/B/E/S coverage (Proport) in a given country-year in our sample is $21.8 \% .^{10}$

Insider trading laws exist (i.e., have been adopted in current or prior years) for $68.7 \%$ of the country-years in our sample, and have been enforced (in the current or prior years) in $23.9 \%$ of the country-years. For $42.1 \%$ of the countryyears in our sample, the capital markets have been liberalized in the current or prior years. Finally, $74.8 \%$ of the country-years observations relate to emerging markets. Our classification of countries into developed and emerging markets and dates of liberalization are detailed in Appendix B.

Table I, Panel B provides a correlation matrix. The measures $\log$ (Analyst) and $\log$ (Proport) are highly related with Pearson correlations of 0.806 . Both

\footnotetext{
${ }^{10}$ Due to the skewness inherent in these analyst coverage variables, we take the log of one plus Analyst and one plus Proport, respectively, and use these log-transformed variables (denoted $\log ($ Analyst $)$ and $\log ($ Proport $)$ ) as our dependent variables. Descriptive statistics for these log-transformed variables are also presented in Table I.
} 


\section{Table I}

Descriptive Statistics

Panel A presents descriptive statistics for country-level analyst coverage and select financial, economic, and insider trading attributes for a sample of 100 countries over the period 1987-2001. Panel B presents Pearson (Spearman) correlations above (below) the diagonal of the matrix for these variables. Two-tailed $p$-values are presented in parentheses. All variables are defined in Appendix A.

\begin{tabular}{|c|c|c|c|c|c|c|}
\hline \multicolumn{7}{|c|}{ Panel A: Descriptive Statistics } \\
\hline & $n$ & Mean & Std. Dev. & Median & Minimum & Maximum \\
\hline Analyst & 1,191 & 2.672 & 3.676 & 0.000 & 0.000 & 17.225 \\
\hline Proport & 1,191 & 0.218 & 0.313 & 0.000 & 0.000 & 1.423 \\
\hline $\log ($ Analyst $)$ & 1,191 & 0.822 & 0.963 & 0.000 & 0.000 & 2.903 \\
\hline $\log ($ Proport $)$ & 1,191 & 0.169 & 0.230 & 0.000 & 0.000 & 0.885 \\
\hline $\log ($ Listed $)$ & 1,191 & 4.735 & 1.721 & 4.920 & 0.000 & 9.088 \\
\hline $\log (G N P)$ & 1,191 & 8.216 & 1.411 & 8.077 & 5.109 & 10.776 \\
\hline Growth $(G N P)$ & 1,191 & 2.125 & 5.332 & 2.377 & -31.811 & 96.374 \\
\hline Emerge & 1,191 & 0.748 & 0.434 & 1.000 & 0.000 & 1.000 \\
\hline Liberal & 1,191 & 0.421 & 0.494 & 0.000 & 0.000 & 1.000 \\
\hline$F D I$ & 1,095 & 1.644 & 2.316 & 0.910 & -6.871 & 17.138 \\
\hline Open & 1,168 & 79.936 & 54.193 & 67.600 & 8.959 & 406.75 \\
\hline Civillaw & 1,191 & 0.514 & 0.500 & 1.000 & 0.000 & 1.000 \\
\hline Socialist & 1,191 & 0.145 & 0.353 & 0.000 & 0.000 & 1.000 \\
\hline Exist & 1,191 & 0.687 & 0.464 & 1.000 & 0.000 & 1.000 \\
\hline Enforce & 1,191 & 0.239 & 0.427 & 0.000 & 0.000 & 1.000 \\
\hline
\end{tabular}


Table I-Continued

\begin{tabular}{|c|c|c|c|c|c|c|c|c|c|c|c|c|c|}
\hline \multicolumn{14}{|c|}{ Panel B: Correlation Matrix } \\
\hline & $\begin{array}{c}\log \\
(\text { Analyst })\end{array}$ & $\begin{array}{c}\log \\
\text { (Proport) }\end{array}$ & $\begin{array}{c}\log \\
(\text { Listed) }\end{array}$ & $\begin{array}{l}\log \\
(G N P)\end{array}$ & $\begin{array}{c}\text { Growth } \\
(G N P)\end{array}$ & Emerge & Liberal & $F D I$ & Open & Civillaw & Socialist & Exist & Enforce \\
\hline $\log ($ Analyst $)$ & $\begin{array}{c}1.000 \\
-\end{array}$ & $\begin{array}{c}0.806 \\
(0.000)\end{array}$ & $\begin{array}{c}0.559 \\
(0.000)\end{array}$ & $\begin{array}{c}0.539 \\
(0.000)\end{array}$ & $\begin{array}{c}0.108 \\
(0.000)\end{array}$ & $\begin{array}{c}-0.644 \\
(0.000)\end{array}$ & $\begin{array}{c}0.745 \\
(0.000)\end{array}$ & $\begin{array}{c}0.125 \\
(0.000)\end{array}$ & $\begin{array}{c}0.073 \\
(0.013)\end{array}$ & $\begin{array}{c}0.099 \\
(0.001)\end{array}$ & $\begin{array}{c}-0.145 \\
(0.000)\end{array}$ & $\begin{array}{c}0.417 \\
(0.000)\end{array}$ & $\begin{array}{c}0.601 \\
(0.000)\end{array}$ \\
\hline $\log ($ Proport $)$ & $\begin{array}{c}0.913 \\
(0.000)\end{array}$ & $\begin{array}{c}1.000 \\
-\end{array}$ & $\begin{array}{c}0.408 \\
(0.000)\end{array}$ & $\begin{array}{c}0.570 \\
(0.000)\end{array}$ & $\begin{array}{c}0.105 \\
(0.000)\end{array}$ & $\begin{array}{c}-0.667 \\
(0.000)\end{array}$ & $\begin{array}{c}0.718 \\
(0.000)\end{array}$ & $\begin{array}{c}0.080 \\
(0.008)\end{array}$ & $\begin{array}{c}0.030 \\
(0.313)\end{array}$ & $\begin{array}{c}0.168 \\
(0.000)\end{array}$ & $\begin{array}{r}-0.172 \\
(0.000)\end{array}$ & $\begin{array}{c}0.348 \\
(0.000)\end{array}$ & $\begin{array}{c}0.507 \\
(0.000)\end{array}$ \\
\hline $\log ($ Listed $)$ & $\begin{array}{c}0.615 \\
(0.000)\end{array}$ & $\begin{array}{c}0.548 \\
(0.000)\end{array}$ & $\begin{array}{c}1.000 \\
-\end{array}$ & $\begin{array}{c}0.272 \\
(0.000)\end{array}$ & $\begin{array}{c}0.135 \\
(0.000)\end{array}$ & $\begin{array}{c}-0.378 \\
(0.000)\end{array}$ & $\begin{array}{c}0.493 \\
(0.000)\end{array}$ & $\begin{array}{c}-0.058 \\
(0.056)\end{array}$ & $\begin{array}{c}-0.172 \\
(0.000)\end{array}$ & $\begin{array}{c}0.085 \\
(0.003)\end{array}$ & $\begin{array}{c}-0.231 \\
(0.000)\end{array}$ & $\begin{array}{c}0.327 \\
(0.000)\end{array}$ & $\begin{array}{c}0.443 \\
(0.000)\end{array}$ \\
\hline $\log (G N P)$ & $\begin{array}{c}0.533 \\
(0.000)\end{array}$ & $\begin{array}{c}0.563 \\
(0.000)\end{array}$ & $\begin{array}{c}0.240 \\
(0.000)\end{array}$ & $\begin{array}{c}1.000 \\
-\end{array}$ & $\begin{array}{c}0.053 \\
(0.070)\end{array}$ & $\begin{array}{c}-0.724 \\
(0.000)\end{array}$ & $\begin{array}{c}0.524 \\
(0.000)\end{array}$ & $\begin{array}{c}0.031 \\
(0.304)\end{array}$ & $\begin{array}{c}0.210 \\
(0.000)\end{array}$ & $\begin{array}{l}0.230 \\
(0.000)\end{array}$ & $\begin{array}{c}-0.214 \\
(0.000)\end{array}$ & $\begin{array}{c}0.244 \\
(0.000)\end{array}$ & $\begin{array}{c}0.426 \\
(0.000)\end{array}$ \\
\hline $\operatorname{Growth}(G N P)$ & $\begin{array}{c}0.138 \\
(0.000)\end{array}$ & $\begin{array}{c}0.142 \\
(0.000)\end{array}$ & $\begin{array}{c}0.118 \\
(0.000)\end{array}$ & $\begin{array}{c}0.017 \\
(0.559)\end{array}$ & $\begin{array}{c}1.000 \\
-\end{array}$ & $\begin{array}{c}-0.044 \\
(0.133)\end{array}$ & $\begin{array}{c}0.085 \\
(0.004)\end{array}$ & $\begin{array}{c}0.073 \\
(0.016)\end{array}$ & $\begin{array}{c}0.099 \\
(0.001)\end{array}$ & $\begin{array}{r}-0.002 \\
(0.935)\end{array}$ & $\begin{array}{c}-0.004 \\
(0.888)\end{array}$ & $\begin{array}{c}0.120 \\
(0.001)\end{array}$ & $\begin{array}{c}0.073 \\
(0.012)\end{array}$ \\
\hline Emerge & $\begin{array}{c}-0.640 \\
(0.000)\end{array}$ & $\begin{array}{c}-0.662 \\
(0.000)\end{array}$ & $\begin{array}{c}-0.384 \\
(0.000)\end{array}$ & $\begin{array}{c}-0.711 \\
(0.000)\end{array}$ & $\begin{array}{c}-0.017 \\
(0.556)\end{array}$ & $\begin{array}{c}1.000 \\
-\end{array}$ & $\begin{array}{c}-0.681 \\
(0.000)\end{array}$ & $\begin{array}{r}-0.060 \\
(0.046)\end{array}$ & $\begin{array}{r}-0.172 \\
(0.000)\end{array}$ & $\begin{array}{c}-0.139 \\
(0.000)\end{array}$ & $\begin{array}{c}0.239 \\
(0.000)\end{array}$ & $\begin{array}{r}-0.196 \\
(0.000)\end{array}$ & $\begin{array}{c}-0.409 \\
(0.000)\end{array}$ \\
\hline Liberal & $\begin{array}{c}0.745 \\
(0.000)\end{array}$ & $\begin{array}{c}0.759 \\
(0.000)\end{array}$ & $\begin{array}{c}0.514 \\
(0.000)\end{array}$ & $\begin{array}{c}0.520 \\
(0.000)\end{array}$ & $\begin{array}{c}0.096 \\
(0.001)\end{array}$ & $\begin{array}{c}-0.681 \\
(0.000)\end{array}$ & $\begin{array}{c}1.000 \\
-\end{array}$ & $\begin{array}{c}0.057 \\
(0.058)\end{array}$ & $\begin{array}{c}0.041 \\
(0.159)\end{array}$ & $\begin{array}{c}0.250 \\
(0.000)\end{array}$ & $\begin{array}{c}-0.351 \\
(0.000)\end{array}$ & $\begin{array}{c}0.289 \\
(0.000)\end{array}$ & $\begin{array}{c}0.411 \\
(0.000)\end{array}$ \\
\hline$F D I$ & $\begin{array}{c}0.150 \\
(0.000)\end{array}$ & $\begin{array}{c}0.110 \\
(0.000)\end{array}$ & $\begin{array}{c}0.014 \\
(0.648)\end{array}$ & $\begin{array}{c}0.048 \\
(0.112)\end{array}$ & $\begin{array}{c}0.031 \\
(0.308)\end{array}$ & $\begin{array}{c}-0.085 \\
(0.005)\end{array}$ & $\begin{array}{c}0.121 \\
(0.000)\end{array}$ & $\begin{array}{c}1.000 \\
-\end{array}$ & $\begin{array}{c}0.394 \\
(0.000)\end{array}$ & $\begin{array}{c}-0.147 \\
(0.000)\end{array}$ & $\begin{array}{c}-0.231 \\
(0.000)\end{array}$ & $\begin{array}{c}0.139 \\
(0.000)\end{array}$ & $\begin{array}{c}0.008 \\
(0.783)\end{array}$ \\
\hline Open & $\begin{array}{c}-0.085 \\
(0.004)\end{array}$ & $\begin{array}{r}-0.110 \\
(0.000)\end{array}$ & $\begin{array}{r}-0.303 \\
(0.000)\end{array}$ & $\begin{array}{c}0.134 \\
(0.000)\end{array}$ & $\begin{array}{c}0.103 \\
(0.000)\end{array}$ & $\begin{array}{r}-0.015 \\
(0.589)\end{array}$ & $\begin{array}{r}-0.124 \\
(0.000)\end{array}$ & $\begin{array}{c}0.189 \\
(0.000)\end{array}$ & $\begin{array}{c}1.000 \\
-\end{array}$ & $\begin{array}{c}0.039 \\
(0.198)\end{array}$ & $\begin{array}{c}0.072 \\
(0.014)\end{array}$ & $\begin{array}{c}0.041 \\
(0.163)\end{array}$ & $\begin{array}{c}-0.084 \\
(0.004)\end{array}$ \\
\hline Civillaw & $\begin{array}{c}0.099 \\
(0.001)\end{array}$ & $\begin{array}{c}0.168 \\
(0.000)\end{array}$ & $\begin{array}{c}0.085 \\
(0.003)\end{array}$ & $\begin{array}{c}0.230 \\
(0.000)\end{array}$ & $\begin{array}{c}-0.002 \\
(0.935)\end{array}$ & $\begin{array}{c}-0.139 \\
(0.000)\end{array}$ & $\begin{array}{c}0.250 \\
(0.000)\end{array}$ & $\begin{array}{c}-0.076 \\
(0.012)\end{array}$ & $\begin{array}{r}-0.236 \\
(0.000)\end{array}$ & $\begin{array}{c}1.000 \\
-\end{array}$ & $\begin{array}{r}-0.424 \\
(0.000)\end{array}$ & $\begin{array}{c}0.017 \\
(0.560)\end{array}$ & $\begin{array}{c}-0.071 \\
(0.014)\end{array}$ \\
\hline Socialist & $\begin{array}{c}-0.145 \\
(0.000)\end{array}$ & $\begin{array}{c}-0.172 \\
(0.000)\end{array}$ & $\begin{array}{c}-0.231 \\
(0.000)\end{array}$ & $\begin{array}{c}-0.214 \\
(0.000)\end{array}$ & $\begin{array}{c}-0.004 \\
(0.888)\end{array}$ & $\begin{array}{c}0.239 \\
(0.000)\end{array}$ & $\begin{array}{c}-0.351 \\
(0.000)\end{array}$ & $\begin{array}{c}0.017 \\
(0.570)\end{array}$ & $\begin{array}{c}0.182 \\
(0.000)\end{array}$ & $\begin{array}{c}-0.424 \\
(0.000)\end{array}$ & $\begin{array}{c}1.000 \\
-\end{array}$ & $\begin{array}{c}0.038 \\
(0.195)\end{array}$ & $\begin{array}{c}-0.092 \\
(0.002)\end{array}$ \\
\hline Exist & $\begin{array}{c}0.416 \\
(0.000)\end{array}$ & $\begin{array}{c}0.394 \\
(0.000)\end{array}$ & $\begin{array}{c}0.316 \\
(0.000)\end{array}$ & $\begin{array}{c}0.264 \\
(0.000)\end{array}$ & $\begin{array}{c}0.150 \\
(0.000)\end{array}$ & $\begin{array}{c}-0.196 \\
(0.000)\end{array}$ & $\begin{array}{c}0.289 \\
(0.000)\end{array}$ & $\begin{array}{c}0.112 \\
(0.000)\end{array}$ & $\begin{array}{c}0.065 \\
(0.026)\end{array}$ & $\begin{array}{c}0.017 \\
(0.560)\end{array}$ & $\begin{array}{c}0.038 \\
(0.195)\end{array}$ & $\begin{array}{c}1.000 \\
-\end{array}$ & $\begin{array}{c}0.378 \\
(0.000)\end{array}$ \\
\hline Enforce & $\begin{array}{c}0.597 \\
(0.000)\end{array}$ & $\begin{array}{c}0.543 \\
(0.000)\end{array}$ & $\begin{array}{c}0.482 \\
(0.000)\end{array}$ & $\begin{array}{c}0.432 \\
(0.000)\end{array}$ & $\begin{array}{c}0.106 \\
(0.006)\end{array}$ & $\begin{array}{c}-0.409 \\
(0.000)\end{array}$ & $\begin{array}{c}0.411 \\
(0.000)\end{array}$ & $\begin{array}{c}0.051 \\
(0.092)\end{array}$ & $\begin{array}{c}0.036 \\
(0.220)\end{array}$ & $\begin{array}{c}-0.071 \\
(0.014)\end{array}$ & $\begin{array}{c}-0.092 \\
(0.002)\end{array}$ & $\begin{array}{c}0.379 \\
(0.000)\end{array}$ & $\begin{array}{l}1.000 \\
-\end{array}$ \\
\hline
\end{tabular}


measures are positively and significantly correlated with the log of the number of listed firms, $\log ($ Listed $)$, with the level and growth in a country's per capita wealth $(\log (G N P)$ and Growth $(G N P))$, with whether a country has liberalized its financial markets (Liberal), and with both the adoption (Exist) and enforcement (Enforce) of insider trading laws. Finally, both measures of analyst following are negatively correlated with whether a country is classified as an emerging market (Emerge).

\section{Research Design and Empirical Results}

This section is organized as follows. Sections III.A and III.B present regressions testing our main hypothesis that analyst following increases upon insider trading law enactment and enforcement, and examine the effect of financial development and financial liberalization on the results. Sections III.C and III.D further examine the robustness of our main results. In Section III.C, we estimate separate regressions for emerging markets and developed economies (in contrast to a pooled model using an emerging market dummy). Section III.D examines the robustness of our results after restricting the sample to the 19922000 time period. Finally, Section III.E extends the empirical analysis to investigate how changes in analyst following upon insider trading restrictions are impacted by countries' preexisting levels of investor protections.

\section{A. Baseline Regressions}

We combine the dates of adoption and enforcement of insider trading laws from Bhattacharya and Daouk (2002) with analyst data from I/B/E/S and other variables to form a panel of annual observations for 100 countries covering the time period 1987-2000. The largest panel contains 1,191 country/year observations. ${ }^{11}$ Our primary specification tests whether analyst activity is higher in countries and years after the enactment (Exist) or initial enforcement (Enforce) of insider trading restrictions, after controlling for global year effects and a variety of other country-level factors. We use a pooled GLS technique to estimate all equations.

Table II reports our baseline regression results using unadjusted analyst data from I/B/E/S (labeled Raw data) and trend-adjusted data (labeled Trendadjusted data). To trend adjust the analyst data, we note that six countries (Canada, France, Singapore, United Kingdom, the United States, and Brazil) enforced insider trading restrictions prior to the start of our sample period (see Appendix B). ${ }^{12}$ As such, these countries exhibit no variation in the treatment variables Enforce or Exist. Since these countries should not influence our test of changing enforcement regimes, we define trend-adjusted analyst coverage variables as the raw level of coverage in country $i$, year $t$ (i.e., $\log \left(\right.$ Analyst $\left._{i, t}\right)$

\footnotetext{
${ }^{11}$ In arriving at 1,191 observations, we delete all country/year observations prior to the creation of a county's stock exchange.

${ }^{12}$ We thank the anonymous referee for suggesting this trend-adjustment procedure.
} 
Table II

\section{Relation between Insider Trading Laws and Analyst Activity}

This panel presents coefficients from various estimations of the following model:

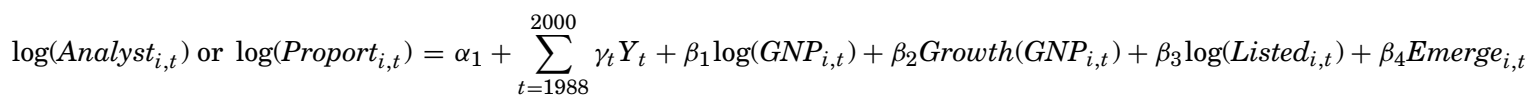

$+\beta_{5}$ Exist $_{i, t}+\beta_{6}$ Enforce $_{i, t}+\beta_{7}$ Exist $_{i, t} *$ Emerge $_{i, t}+\beta_{8}$ Enforce $_{i, t} *$ Emerge $_{i, t}+\varepsilon_{i, t}$.

The models are estimated on a panel of countries with a maximum of 14 time-series observations (calendar years 1987 through 2000 ) using a pooled GLS estimation technique with annual indicator variables $\left(Y_{t}\right)$. Trend-adjusted data are defined as the raw level of analyst coverage in country $i$, year $t$ less the average level of coverage reported for six countries that enforced insider trading restrictions prior to 1987 in the same calendar year Two-tailed $p$-values are presented in parentheses. Bootstrapped $p$-values (one-tail) for the coefficients related to Enforce are presented in italics and parentheses below the GLS-derived $p$-values. All variables are defined in Appendix A.

\begin{tabular}{cccccccc}
\hline $\log ($ GNP $)$ & Growth $($ GNP $)$ & $\log ($ Listed $)$ & Emerge & Exist & Enforce & Exist $*$ Emerge & Enforce $*$ Emerge \\
\hline Panel A: Emerging versus Developed Economies
\end{tabular}

Dependent Variable: Number of Analysts Following I/B/E/S Firms: $\log \left(\right.$ Analyst $\left._{i, t}\right)$

Raw data (100 countries)

$0.394 \quad-0.001$

$\begin{array}{cc}0.394 & -0.001 \\ (0.000) & (0.689)\end{array}$

$\begin{array}{ccc}0.156 & -0.175 & -0.010 \\ (0.000) & (0.415) & (0.783) \\ - & - & - \\ 0.141 & -0.471 & -0.005 \\ (0.000) & (0.023) & (0.939)\end{array}$

0.325

(0.000)

$0 . \overline{351}$

$-0.001$

(0.000)

(0.757)

(0.000)

$-$

$-$

(0.002)

0.068

(0.263)

(0.138)
$-$
$-$
$-\overline{-} 006$
(0.938)

$-$

0.2747

1191

-

Trend-adjusted data (94 countries)

\begin{tabular}{|c|c|c|c|c|c|c|c|c|c|}
\hline 0.410 & -0.002 & 0.162 & -0.158 & -0.024 & 0.308 & - & - & 0.2717 & 1107 \\
\hline$(0.000)$ & $(0.446)$ & $(0.000)$ & $(0.508)$ & $(0.536)$ & $(0.000)$ & - & - & & \\
\hline- & - & - & - & - & $(0.004)$ & - & - & & \\
\hline $\begin{array}{c}0.367 \\
(0.000)\end{array}$ & $\begin{array}{c}-0.001 \\
(0.502)\end{array}$ & $\begin{array}{c}0.147 \\
(0.000)\end{array}$ & $\begin{array}{r}-0.406 \\
(0.081)\end{array}$ & $\begin{array}{r}-0.013 \\
(0.844)\end{array}$ & $\begin{array}{c}0.054 \\
(0.375)\end{array}$ & $\begin{array}{c}-0.001 \\
(0.989)\end{array}$ & $\begin{array}{r}0.463^{\mathrm{a}} \\
(0.000)\end{array}$ & 0.2999 & 1107 \\
\hline- & - & - & - & - & $(0.276)$ & - & $(0.048)^{\mathrm{a}}$ & & \\
\hline
\end{tabular}


Raw data

0.078

Dependent Variable: Proportion of I/B/E/S Covered Firms to Domestic Listed Firms: $\log \left(\right.$ Proport $\left._{i, t}\right)$

$(0.000)$

0.001

0.001
$(0.211)$

0.009

-0.153
$(0.002)$

-0.015
$(0.113)$

0.041

$(0.000)$

0.001

0.074

$(0.000)$

$(0.190)$

(0.002)

$(0.068)$
-0.012

0.005

$-0.231$

$-0.041$

(0.001)

(0.018)

$(0.452)$

(0.416)

-
-
-
0.036
$(0.060)$

$-$

0.1554

1191

Trend-adjusted data

0.082

0.001

$-$

$-$

$-$

0.060

$0.101^{\mathrm{a}}$
$(0.000)$
$(0.100)^{\mathrm{a}}$

(0.000)

(0.318)

0.011

$-0.165$

(0.003)

$(0.003)$

$-0.019$

0.038

$0.054)$

$(0.001)$

(0.090)

0.006

-
-0.233

-
-0.045

0.001

(0.083)

(0.000)

(0.010)

(0.403)

(0.510)

-
-
-
0.036
$(0.060)$
-

-
-
-
$0.098^{\mathrm{a}}$
$(0.000)$

$(0.094)^{\mathrm{a}}$

(continued) 
Table II-Continued

This panel presents coefficients from various estimations of the following model:

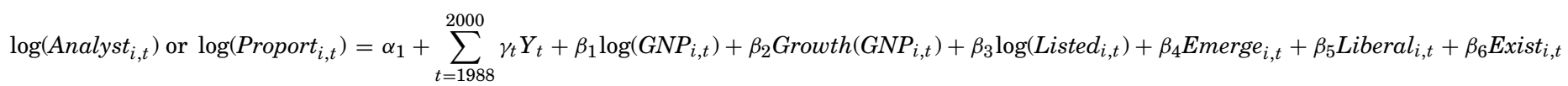

$$
+\beta_{7} \text { Enforce }_{i, t}+\beta_{8} \text { Exist }_{i, t} * \text { Liberal }_{i, t}+\beta_{9} \text { Enforce }_{i, t} * \operatorname{Liberal}_{i, t}+\varepsilon_{i, t} .
$$

The models are estimated on a panel of countries with a maximum of 14 time-series observations (calendar years 1987 through 2000 ) using a pooled GLS estimation technique with annual indicator variables $\left(Y_{t}\right)$. Trend-adjusted data is defined as the raw level of analyst coverage in country $i$, year $t$ less the average level of coverage reported for six countries that enforced insider trading restrictions prior to 1987 in the same calendar year. Two-tailed $p$-values are presented in parentheses. Bootstrapped $p$-values (one-tail) for the coefficients related to Enforce are presented in italics and parentheses below the GLS-derived $p$-values. All variables are defined in Appendix A.

\begin{tabular}{|c|c|c|c|c|c|c|c|c|c|c|}
\hline \multicolumn{11}{|c|}{ Panel B: Role of Financial Markets Liberalization } \\
\hline $\log (G N P)$ & $\operatorname{Grow}(G N P)$ & $\log ($ Listed $)$ & Emerge & Liberal & Exist & Enforce & Exist* Liberal & Enforce $*$ Liberal & Adj. $R^{2}$ & $n$ \\
\hline \multicolumn{11}{|c|}{ Dependent Variable: Number of Analysts Following I/B/E/S Firms: $\log \left(\right.$ Analyst $\left._{i, t}\right)$} \\
\hline \multicolumn{11}{|c|}{ Raw data (100 countries) } \\
\hline 0.423 & -0.001 & 0.154 & 0.338 & 0.512 & 0.023 & 0.348 & - & - & 0.3266 & 1191 \\
\hline$(0.000)$ & $(0.627)$ & $(0.000)$ & $(0.139)$ & $(0.000)$ & $(0.532)$ & $(0.000)$ & - & - & & \\
\hline- & - & - & - & - & - & $(0.000)$ & - & - & & \\
\hline 0.363 & -0.001 & 0.155 & 0.195 & 0.354 & -0.091 & 0.511 & $0.313^{\mathrm{d}}$ & $-0.268^{\mathrm{d}}$ & 0.3445 & 1191 \\
\hline$(0.000)$ & $(0.604)$ & $(0.000)$ & $(0.362)$ & $(0.000)$ & $(0.031)$ & $(0.000)$ & $(0.000)$ & $(0.002)$ & & \\
\hline- & - & - & - & - & - & $(0.000)$ & - & $(0.052)$ & & \\
\hline \multicolumn{11}{|c|}{ Trend-adjusted data (94 countries) } \\
\hline 0.424 & -0.002 & 0.160 & 0.250 & 0.428 & 0.009 & 0.333 & - & - & 0.3057 & 1107 \\
\hline$(0.000)$ & $(0.442)$ & $(0.000)$ & $(0.310)$ & $(0.000)$ & $(0.807)$ & $(0.000)$ & - & - & & \\
\hline- & - & - & - & - & - & $(0.000)$ & - & - & & \\
\hline $\begin{array}{c}0.360 \\
(0.000)\end{array}$ & $\begin{array}{c}-0.002 \\
(0.329)\end{array}$ & $\begin{array}{c}0.157 \\
(0.000)\end{array}$ & $\begin{array}{c}0.081 \\
(0.724)\end{array}$ & $\begin{array}{c}0.278 \\
(0.000)\end{array}$ & $\begin{array}{c}-0.108 \\
(0.011)\end{array}$ & $\begin{array}{c}0.597 \\
(0.000)\end{array}$ & $\begin{array}{c}0.314^{\mathrm{d}} \\
(0.000)\end{array}$ & $\begin{array}{r}-0.408^{\mathrm{d}} \\
(0.000)\end{array}$ & 0.3339 & 1107 \\
\hline - & - & - & - & - & - & $(0.000)$ & - & $(0.000)$ & & \\
\hline
\end{tabular}


Dependent Variable: Proportion of I/B/E/S Covered Firms to Domestic Listed Firms: $\log \left(\right.$ Proport $\left._{i, t}\right)$

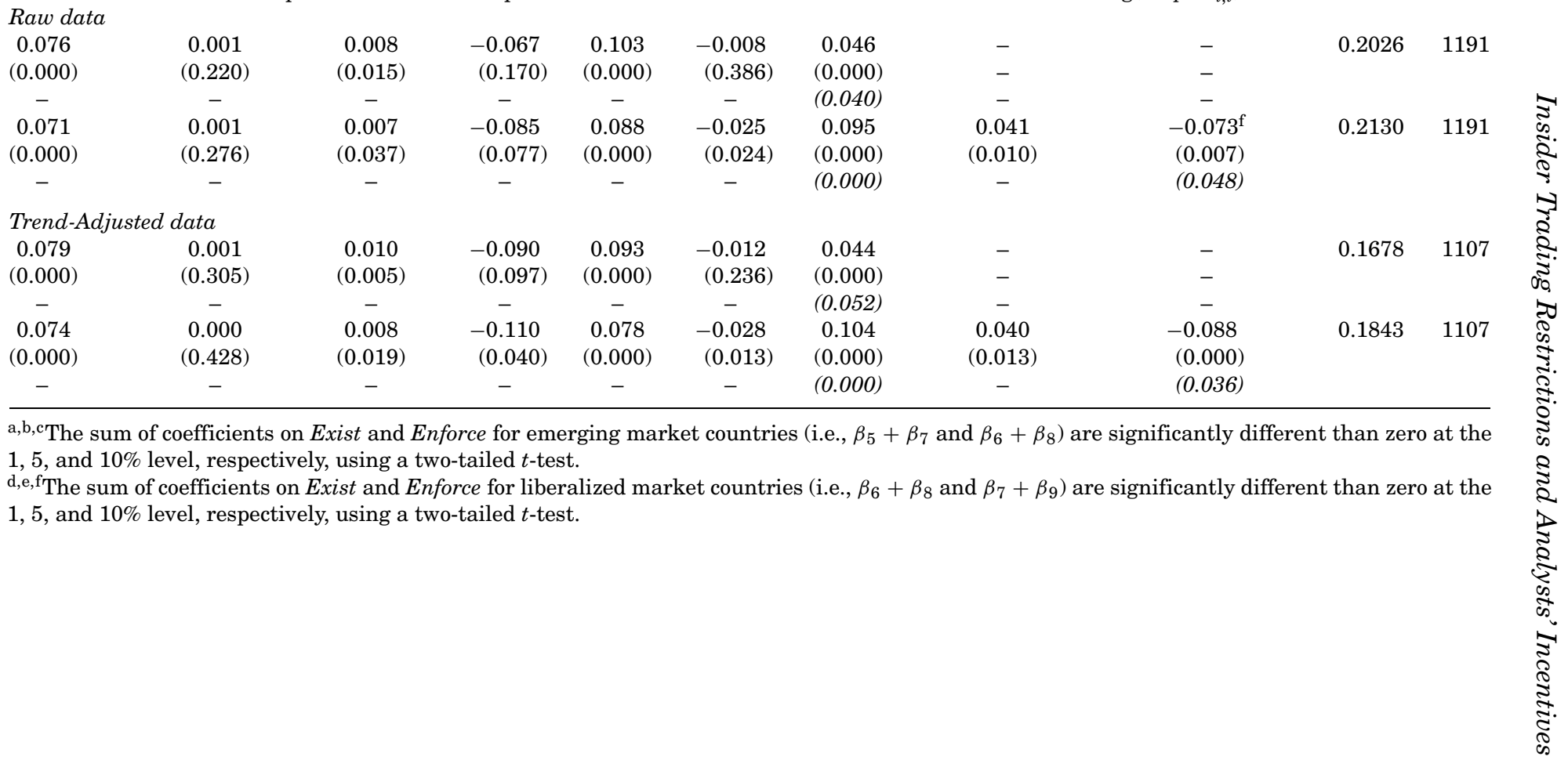


and $\log \left(\right.$ Proport $\left.\left._{i, t}\right)\right)$ less the average level of coverage reported for these six control countries in the same calendar year. ${ }^{13}$

In addition to the potential for our estimated coefficients to capture trends or macro-effects in the data other than effects associated with insider trading restrictions, GLS $p$-values could also potentially be biased due to cross-sectional and time-series dependencies in the data. To test the robustness to these issues of our main result, a positive relation between analyst coverage and Enforce, we bootstrap the statistical significance of our enforcement coefficients using the following procedure.

First, we randomly assign our sample countries into two groups: those countries that did enforce and those countries that did not enforce insider trading restrictions over our sample period. This assignment is in the same proportion as the sample countries in the actual data. For those firms that were randomly chosen to have enforced insider trading restrictions, we next randomly assigned an initial year of enforcement between 1987 and 2000, and define all enforcement indicator variables accordingly. Next, we estimate our multivariate models using these random country-event dates and retain the estimated coefficients on our enforcement variables. We repeat this random assignment and estimation procedure 500 times, yielding an empirical distribution of enforcement-related coefficients under the null of no relation between insider trading restrictions and analyst coverage. We use this empirical distribution under the null to test the statistical significance of the coefficients from our estimations using the actual panel data. One-tail bootstrap $p$-values are presented for Enforce in italics and parentheses below our GLS-derived $p$-values in Table II.

In Table II, Panel A, our estimations include four control variables (and year indicators) in addition to the insider trading variables. We include $\log (G N P)$ as the measure of a country's per-capita wealth, and annual growth in real GNP per capita (Growth $(G N P))$. Results indicate that both measures of analyst following are significantly higher in wealthy countries. We also control for the size of the stock market as measured by the number of domestic firms listed on the home country's exchange, $\log ($ Listed $)$, and find that analyst following is higher for countries with more listed firms. Finally, we also include an indicator variable Emerge equal to 1 if the country is classified as an emerging market, and 0 otherwise.

In terms of insider trading restrictions, our estimations reveal that Exist is either unrelated or significantly negatively related to both measures of analyst following, while Enforce is significantly positively related to both analyst measures in the regression models without interaction variables. Thus, there is an increase in analyst following upon the enforcement of insider trading laws, but

\footnotetext{
${ }^{13}$ To estimate the trend adjustment, we measured annual average coverage in our six control countries using equal-weighted observations and a value-weighted average where observations are weighted by the level of the country's coverage. Results using these two trend-adjustments are similar. For parsimony, we only report estimations using the trend adjustment based on equalweighted averages.
} 
no increase upon the enactment of these laws. ${ }^{14}$ This result holds using both raw and trend-adjusted data, and is robust to our bootstrap procedures. For Analyst, bootstrapped $p$-values for Enforce are 0.002 (0.004) using raw (trendadjusted) data, and for Proport, bootstrapped $p$-values for Enforce are 0.068 (0.090) for raw (trend-adjusted) data. The observed increase in analyst following upon the enforcement, but not the enactment of insider trading restrictions, is consistent with the findings of Bhattacharya and Daouk (2002) relative to cost of equity capital.

The second set of estimations presented in Table II, Panel A includes interaction terms to search for a differential response to insider trading laws across emerging and developed markets. These estimations indicate that the sensitivity of analyst following to the enforcement variable is significantly stronger in emerging markets than in developed markets, as reflected by the positive and significant coefficient on the interaction term Enforce $*$ Emerge for both analyst following variables $\left(\beta_{8}>0\right)$. This result holds using both raw and trendadjusted data, with bootstrapped $p$-values ranging from 0.048 to 0.100 .

In these estimations, the coefficient on Enforce $\left(\beta_{6}\right)$ represents the change in analyst following associated with the initial enforcement of insider trading restrictions in the subsample of developed countries, after controlling for other country-level attributes and global year effects. This coefficient is not significantly greater than zero for either analyst measure. In contrast, the coefficient on Enforce for the subsample of emerging market countries, which is represented by the sum $\beta_{6}+\beta_{8}$ (i.e., the coefficient on Enforce plus the interaction term Enforce $*$ Emerge), is significantly positive at the $1 \%$ level for both measures of analyst coverage. ${ }^{15}$ This statistical significance is indicated by the superscript "a" on the coefficient on Enforce $*$ Emerge ${ }^{16}$ Similarly, the superscript "a" on the bootstrapped $p$-values for Enforce $*$ Emerge indicates that the same significance level is achieved for the sum $\beta_{6}+\beta_{8}$ using our empirically derived distribution of coefficients. Together, this evidence suggests that the enforcement effect is concentrated in emerging market countries.

Table II, Panel B extends the analysis to control for financial market liberalization (data source: Bekaert et al. 2001). Liberalization of equity markets is a potentially profound policy reform that opens a country's market to the free flow of capital. Liberalization can result in an inflow of foreign capital, which can improve risk sharing and pressure firms to improve governance. A number of other macro-economic reforms may also occur simultaneously with liberalization. We therefore want to distinguish the

${ }^{14}$ In terms of economic significance, note that, holding all other variables constant across Enforce $=1$ or Enforce $=0$, $(1+$ predicted value Analyst $\mid$ Enforce $=1) /(1+$ predicted value of Analyst $\mid$ Enforce $=0)=\exp \left(\right.$ coefficient on Enforce $=\beta_{6}$ ). Thus for Analyst, Table II, Panel A indicates $\exp (0.325)=1.38$. Similarly for Proport, we get $\exp (0.041)=1.04$.

${ }^{15}$ Analogously, the coefficient on Exist for the emerging markets subsample is defined by $\beta_{5}+$ $\beta_{7}$.

${ }^{16}$ Whereas the numerical $p$-values reported in the tables relate to the individual estimated coefficients, we indicate the significance of sums of coefficients (if they are significant) with superscripts reported next to the coefficient on the interaction variables (e.g., on $\beta_{8}$ in Table II, Panel A). 
liberalization event from insider trading restrictions. The estimations presented in Table II, Panel B are identical to those in Table II, Panel A except that we introduce liberalization (Liberal) into the model and interact Exist and Enforce with Liberal instead of with Emerge. While Liberal and Emerge are negatively correlated $(-0.691)$, the correlation is not perfect. Thus, we explore the extent to which the association between analyst following and insider trading restrictions differs across liberalized and nonliberalized markets.

The results in Table II, Panel B reveal that both measures of analyst following are significantly higher in years following financial market liberalization. However, even after controlling for liberalization, Enforce remains significantly positively related to both analyst measures (both raw and trend-adjusted data, using both GLS and bootstrap-derived $p$-values).

After adding interaction terms, we find a significant negative relation between Exist and both measures of analyst following for the subsample of nonliberalized countries $\left(\beta_{6}<0\right)$. In contrast, for the subsample of liberalized countries, the relation between Exist and analyst following (i.e., $\beta_{6}+\beta_{8}$ ) is positive for both measures of analyst following, and significantly greater than zero for Analyst. In terms of enforcement effects, the relation between Enforce and both measures of analyst following is significantly greater than zero for the nonliberalized subsample (i.e., $\beta_{7}>0$ ). However, this effect is significantly lower for liberalized countries $\left(\beta_{9}<0\right)$, yet the net effect is still marginally positive (i.e., $\beta_{7}+\beta_{9}>0$ ). These results hold for raw and trend-adjusted data, and are robust to our bootstrap procedures.

As defined, our liberalization variable measures the year that a country implements financial market liberalization policies. However, actual financial flows resulting from the act of liberalization may be the true cause of both insider trading restrictions and analyst following, and these flow responses can vary significantly across countries due to country characteristics that we have not controlled for. To mitigate concerns about the issue of correlated omitted variables, we reestimate our models after including two direct measures of country-level financial flows: foreign direct investment (FDI) and trade openness (Open). We find that our main results are robust to the inclusion of foreign direct investment and trade openness.

To summarize, Table II documents that analyst following increases after enforcement of insider trading laws. Panel A documents that the positive relation between insider trading law enforcement and analyst following is significantly higher in emerging markets than in developed countries, with no similar effect for enactment of the law. Panel B documents that the coefficient on the enactment of the law is significantly higher for countries that have previously liberalized, while the coefficient on the enforcement of the law is significantly lower for countries that have liberalized relative to those that have not.

This evidence is consistent with a conjecture by Laeven and Perotti (2001), who argue that announced market-oriented policies may be subsequently reversed and so are not initially credible. They find that sustained progress in privatization gradually leads to increased public confidence, which in turn has a strong effect on market development. In emerging markets, enforcement of 
insider trading restrictions may signal political commitment and encourage market participants, like analysts, to transition to a new property rights regime, which they are skeptical about at the time of enactment. Similarly, in countries that have already liberalized, enactment of insider trading laws may have relatively more credibility, supporting a strong analyst response at that time and diminishing the importance of initial enforcement as a signal of commitment to change.

\section{B. Change in Analyst Activity in the Year of Enactment and Enforcement}

To reinforce the preceding inferences, we estimate regressions focusing on annual changes in analyst activity around our insider trading events. The variable measuring change in existence of insider trading laws in a given country ( $\Delta E x i s t$ ) is set equal to 1 in the year in which the insider trading laws were enacted in that country, and is set equal to 0 in all other years. Similarly, the variable measuring change in the enforcement regime ( $\Delta$ Enforce) is set equal to 1 for a given country in the year of the initial enforcement of insider trading laws, and is set equal to 0 in all other years. This change regression model specification represents a test of whether the change in analyst activity is higher in the year the insider trading laws were enacted or enforced relative to the surrounding years.

Table III, Panel A presents descriptive statistics on annual changes in analyst coverage over our sample period. Specifically, the average annual change in the number of analysts following covered firms in a country is 0.188 analysts per firm, while the average annual change in the proportion of firms in the country being covered is 0.008 . This increase in analyst coverage is consistent with the observed growth in capital markets over this time frame. In terms of observed insider trading events, we find that 54 countries enacted insider trading restrictions between 1987 and 2000, while 30 countries initially enforced these laws.

Table III, Panel B presents the results of estimations examining changes in analyst coverage over our sample period. ${ }^{17}$ The results are consistent with the inferences drawn from the levels regressions reported in Table II indicating a positive relation between change in analyst following and the enforcement of insider trading restrictions. Before considering interactions, we find that $\triangle$ Analyst is significantly higher in the year of initial enforcement of insider trading laws, although we do not find a significant relation between the change in breadth of analyst following $(\Delta$ Proport) and $\Delta$ Enforce. Also consistent with Table II, for both $\triangle$ Analyst and $\Delta$ Proport, the interaction terms show that the increase in analyst following upon enforcement is significantly higher in emerging market countries and significantly lower in countries that have already liberalized their markets. These results hold for raw and trend-adjusted data and after implementing our bootstrapping procedures. Finally, none of the

\footnotetext{
${ }^{17}$ For ease of presentation and without loss of information, we only tabulate results using trendadjusted data in this and all subsequent tables.
} 


\section{Table III}

\section{Change in Analyst Coverage around the Enactment and Enforcement of Insider Trading Laws}

Panel A presents descriptive statistics for annual changes in analyst coverage in our sample. Panel B presents select coefficients from various estimations of the following model:

$$
\begin{aligned}
\Delta \log \left(\text { Analyst }_{i, t}\right) \text { or } \Delta \log \left(\text { Proport }_{i, t}\right)= & \alpha_{1}+\sum_{t=1989}^{2000} \gamma_{t} Y_{t}+\beta_{1} \log \left(\text { GNP }_{i, t-1}\right)+\beta_{2} \text { Growth }_{\left(G N P_{i, t}\right)} \\
& +\beta_{3} \Delta \log \left(\text { Listed }_{i, t}\right)+\beta_{4} \text { Emerge }_{i, t}+\beta_{5} \text { Liberal }_{i, t} \\
& +\beta_{6} \Delta \text { Exist }_{i, t}+\beta_{7} \Delta \text { Enforce }_{i, t}+\beta_{8} \Delta \text { Exist }_{i, t} * \text { Emerge }_{i, t} \\
& +\beta_{9} \Delta \text { Enforce }_{i, t} * \text { Emerge }_{i, t}+\beta_{10} \Delta \text { Exist }_{i, t} * \text { Liberal }_{i, t} \\
& +\beta_{11} \Delta \text { Enforce }_{i, t} * \text { Liberal }_{i, t}+\varepsilon_{i, t} .
\end{aligned}
$$

The models are estimated on a panel of countries with a minimum (maximum) of 2 (13) time-series observations (i.e., annual changes between calendar years 1987 through 2000) using a pooled GLS estimation technique with annual indicator variables $\left(Y_{t}\right)$. All models are estimated using trend-

\begin{tabular}{|c|c|c|c|c|c|c|c|c|}
\hline \multicolumn{9}{|c|}{ Panel A: Descriptive Statistics } \\
\hline & & $n$ & Mean & Std. Dev. & Median & \multicolumn{2}{|c|}{ Minimum } & Maximum \\
\hline$\triangle$ Analys & & 1,085 & 0.188 & 0.909 & 0.000 & \multicolumn{2}{|c|}{-3.892} & 5.422 \\
\hline$\Delta$ Propor & & 1,085 & 0.008 & 0.103 & 0.000 & \multicolumn{2}{|c|}{-1.025} & 1.056 \\
\hline$\Delta \log (A n$ & $y s t)$ & 1,085 & 0.056 & 0.234 & 0.000 & \multicolumn{2}{|c|}{-0.875} & 1.860 \\
\hline$\Delta \log (\operatorname{Pro}$ & ort) & 1,085 & 0.006 & 0.071 & 0.000 & \multicolumn{2}{|c|}{-0.690} & 0.721 \\
\hline \multicolumn{9}{|c|}{ Panel B: Impact in Year of Change } \\
\hline Emerge & Liberal & $\Delta$ Exist & $\Delta$ Enforce & $\begin{array}{l}\Delta \text { Exist } * \\
\text { Emerge }\end{array}$ & $\begin{array}{l}\Delta \text { Enforce } * \\
\text { Emerge }\end{array}$ & $\begin{array}{l}\Delta \text { Exist } * \\
\text { Lliberal }\end{array}$ & $\begin{array}{l}\Delta \text { Enforce } * \\
\text { Liberal }\end{array}$ & $R^{2}$ \\
\hline \multicolumn{9}{|c|}{$\begin{array}{c}\text { Dependent Variable: Change in Number of Analysts } \\
\text { Following I/B/E/S Firms: } \Delta \log \left(\text { Analyst }_{i, t}\right)\end{array}$} \\
\hline 0.069 & 0.064 & 0.010 & 0.106 & - & - & - & - & 0.0412 \\
\hline$(0.017)$ & $(0.001)$ & $(0.753)$ & $(0.016)$ & - & - & - & - & \\
\hline- & - & - & $(0.050)$ & - & - & - & - & \\
\hline 0.070 & 0.063 & 0.055 & 0.086 & -0.061 & $0.036^{\mathrm{b}}$ & - & - & 0.0420 \\
\hline$(0.018)$ & $(0.001)$ & $(0.384)$ & $(0.201)$ & $(0.406)$ & $(0.681)$ & - & - & \\
\hline- & - & - & $(0.148)$ & - & $(0.292)^{\mathrm{c}}$ & - & - & \\
\hline 0.067 & 0.071 & 0.001 & 0.361 & - & - & 0.022 & -0.371 & 0.0560 \\
\hline$(0.021)$ & $(0.000)$ & $(0.972)$ & $(0.000)$ & - & - & $(0.739)$ & $(0.000)$ & \\
\hline- & - & - & $(0.002)$ & - & - & - & $(0.000)$ & \\
\hline \multicolumn{9}{|c|}{$\begin{array}{l}\text { Dependent Variable: Change in Proportion of I/B/E/S Covered } \\
\text { Firms to Domestic Listed Firms: } \Delta \log \left(\text { Proport }_{i, t}\right)\end{array}$} \\
\hline 0.009 & 0.009 & -0.004 & 0.013 & - & - & - & - & 0.0400 \\
\hline$(0.556)$ & $(0.302)$ & $(0.685)$ & $(0.309)$ & - & - & - & - & \\
\hline- & - & - & $(0.136)$ & - & - & - & - & \\
\hline 0.004 & 0.009 & -0.018 & -0.031 & 0.018 & $0.079^{\mathrm{a}}$ & - & - & 0.0486 \\
\hline$(0.790)$ & $(0.322)$ & $(0.341)$ & $(0.114)$ & $(0.398)$ & $(0.003)$ & - & - & \\
\hline- & - & - & $(0.168)$ & - & $(0.028)^{\mathrm{b}}$ & - & - & \\
\hline 0.009 & 0.012 & 0.002 & 0.099 & - & - & -0.014 & -0.123 & 0.0566 \\
\hline$(0.480)$ & $(0.139)$ & $(0.882)$ & $(0.000)$ & - & - & $(0.475)$ & $(0.000)$ & \\
\hline- & - & - & $(0.000)$ & - & - & - & $(0.000)$ & \\
\hline
\end{tabular}
adjusted analyst coverage data. Two-tailed GLS $p$-values are presented in parentheses below the estimated coefficients. Bootstrapped $p$-values (one-tail) for the coefficients related to Enforce are presented in italics and parentheses below the GLS-derived $p$-values. All variables are defined in Appendix A. $n=1,085$.

a,b,c The sum of coefficients on $\Delta$ Exist and $\Delta$ Enforce for emerging market countries (i.e., $\beta_{6}+\beta_{8}$ and $\beta_{7}+\beta_{9}$ ) and liberalized countries (i.e., $\beta_{6}+\beta_{10}$ and $\beta_{7}+\beta_{11}$ ) are significantly different than zero at the 1,5 , and $10 \%$ level, respectively, using a two-tailed $t$-test. 
coefficients on $\Delta$ Exist or on the interactions of $\Delta$ Exist with Emerge or with Liberal are significant. These results fail to provide any evidence that the change in analyst following in the year that insider trading laws are enacted is higher than in other years.

\section{Separate Regressions for Emerging Markets and Developed Economies}

In Table IV, we estimate separate regressions for emerging markets and developed economies, in contrast to our use of an emerging market dummy

\section{Table IV}

\section{Relation between Insider Trading Laws and Analyst Activity using Separate Estimations for Developed and Emerging Economies}

This table presents select coefficients from the estimation of equation (2) (Panel A) and equation (3) (Panel B) on separate panels of emerging and developed countries, respectively, with a minimum (maximum) of 2 (14 annual or 13 annual changes) time-series observations (calendar years 1987 through 2000) using a pooled GLS estimation technique with annual indicator variables $\left(Y_{t}\right)$. The emerging markets (developed economies) models are estimated for 77 (17) countries using trendadjusted analyst coverage data. Models for the developed economies are estimated after excluding all Liberal terms. Two-tailed $p$-values are presented in parentheses. All variables are defined in Appendix A. The superscript "a" denotes that the sum of coefficients on Exist and Enforce for liberalized market countries (i.e., $\beta_{5}+\beta_{7}$ and $\beta_{6}+\beta_{8}$ ) are significantly different than zero at the $1 \%$ level using a two-tailed $t$-test.

Panel A: Baseline Levels Regressions

\begin{tabular}{|c|c|c|c|c|c|c|}
\hline \multirow{3}{*}{$\begin{array}{l}\text { Dependent Variable } \\
\text { Exist }\end{array}$} & \multicolumn{4}{|c|}{ Emerging Markets $(n=877)$} & \multicolumn{2}{|c|}{ Developed Economies $(n=230)$} \\
\hline & \multicolumn{2}{|c|}{$\log \left(\right.$ Analyst $\left._{i, t}\right)$} & \multicolumn{2}{|c|}{$\log \left(\right.$ Proport $\left._{i, t}\right)$} & \multirow{2}{*}{$\begin{array}{c}\log \left(\text { Analyst }_{i, t}\right) \\
-0.129 \\
(0.020)\end{array}$} & \multirow{2}{*}{$\begin{array}{c}\log \left(\text { Proport }_{i, t}\right) \\
-0.067 \\
(0.004)\end{array}$} \\
\hline & $\begin{array}{c}0.019 \\
(0.668)\end{array}$ & $\begin{array}{r}-0.078 \\
(0.079)\end{array}$ & $\begin{array}{c}-0.001 \\
(0.942)\end{array}$ & $\begin{array}{r}-0.020 \\
(0.073)\end{array}$ & & \\
\hline Enforce & $\begin{array}{c}0.514 \\
(0.000)\end{array}$ & $\begin{array}{c}0.624 \\
(0.000)\end{array}$ & $\begin{array}{c}0.090 \\
(0.000)\end{array}$ & $\begin{array}{c}0.111 \\
(0.000)\end{array}$ & $\begin{array}{c}0.072 \\
(0.140)\end{array}$ & $\begin{array}{c}-0.016 \\
(0.422)\end{array}$ \\
\hline Exist $*$ Liberal & $\begin{array}{l}- \\
-\end{array}$ & $\begin{array}{c}0.682^{\mathrm{a}} \\
(0.000)\end{array}$ & $\begin{array}{l}- \\
-\end{array}$ & $\begin{array}{c}0.138^{\mathrm{a}} \\
(0.000)\end{array}$ & $\begin{array}{l}- \\
-\end{array}$ & $\begin{array}{l}- \\
-\end{array}$ \\
\hline Enforce $*$ Liberal & $\begin{array}{l}- \\
-\end{array}$ & $\begin{array}{r}-0.268^{a} \\
(0.003)\end{array}$ & $\begin{array}{l}- \\
-\end{array}$ & $\begin{array}{r}-0.051^{\mathrm{a}} \\
(0.024)\end{array}$ & $\begin{array}{l}- \\
-\end{array}$ & $\begin{array}{l}- \\
-\end{array}$ \\
\hline$R^{2}$ & 0.3214 & 0.3624 & 0.1628 & 0.1985 & 0.1394 & 0.1729 \\
\hline
\end{tabular}

Panel B: Impact in Year of Change

\begin{tabular}{|c|c|c|c|c|c|c|}
\hline \multirow{4}{*}{$\begin{array}{l}\text { Dependent Variable } \\
\Delta \text { Exist }\end{array}$} & \multicolumn{4}{|c|}{ Emerging Markets $(n=794)$} & \multicolumn{2}{|c|}{ Developed Economies $(n=213)$} \\
\hline & \multicolumn{2}{|c|}{$\Delta \log \left(\right.$ Analyst $\left._{i, t}\right)$} & \multicolumn{2}{|c|}{$\Delta \log \left(\right.$ Proport $\left._{i, t}\right)$} & \multirow{3}{*}{$\begin{array}{c}\Delta \log \left(\text { Analyst }_{i, t}\right) \\
-0.007 \\
(0.860)\end{array}$} & \multirow{3}{*}{$\begin{array}{c}\Delta \log \left(\text { Proport }_{i, t}\right) \\
-0.013 \\
(0.416)\end{array}$} \\
\hline & -0.001 & 0.007 & 0.002 & 0.002 & & \\
\hline & $(0.984)$ & $(0.879)$ & $(0.867)$ & $(0.849)$ & & \\
\hline \multirow[t]{2}{*}{$\Delta$ Enforce } & 0.124 & 0.362 & 0.050 & 0.103 & 0.076 & -0.010 \\
\hline & $(0.046)$ & $(0.000)$ & $(0.005)$ & $(0.000)$ & $(0.068)$ & $(0.551)$ \\
\hline \multirow[t]{2}{*}{$\Delta$ Exist $*$ Liberal } & - & -0.046 & - & 0.003 & - & - \\
\hline & - & $(0.683)$ & - & $(0.937)$ & - & - \\
\hline \multirow[t]{2}{*}{$\Delta$ Enforce $*$ Liberal } & - & -0.522 & - & -0.112 & - & - \\
\hline & - & $(0.000)$ & - & $(0.001)$ & - & - \\
\hline$R^{2}$ & 0.0459 & 0.0671 & 0.0429 & 0.0550 & 0.0985 & 0.3404 \\
\hline
\end{tabular}


(Emerge) in Tables II and III. Panel A reports results from levels regressions and Panel B reports results from a changes specification. This analysis extends Tables II and III in several important respects.

First, this partition of the sample allows us to more cleanly isolate the impact of liberalization. In particular, note from Appendix B that all developed countries liberalized their markets prior to 1987, implying that all liberalization events during our sample window relate to emerging market countries. Second, this specification allows us to split our control countries into separate developed and emerging market benchmarks when trend adjusting our analyst coverage data. Specifically, we measure average analyst coverage each year for the respective set of developed or emerging market control countries to create trend adjustments by type of economy, and match accordingly. ${ }^{18}$ Finally, by estimating these models separately, we allow the coefficients on all country-level control variables and annual indicators to vary across these two fundamentally different sets of countries.

The evidence in Table IV, Panels A and B strongly reinforces the main results reported previously in Tables II and III. Both Panel A (levels specification) and Panel B (changes specification) document that the coefficient on Enforce ( $\triangle$ Enforce) for both Analyst ( $\triangle$ Analyst) and Proport ( $\triangle$ Proport) is significantly positive for emerging market countries and is either insignificant or possesses a smaller coefficient for developed countries. Moreover, these estimations document that within the subsample of emerging market countries, the coefficients on Enforce and $\Delta E$ Eforce for both measures of analyst following are smaller in countries that have liberalized relative to those that have not.

Finally, recall that Table II, Panel B documents that the coefficient on Exist is significantly higher for countries that have liberalized. Table IV, Panel A (levels specification) does document that the coefficient on Exist is higher in countries that have liberalized (the coefficient on Exist $*$ Liberal $>0$ ); however, this result is not supported by the change specification in Panel B (the coefficient on $\Delta$ Exist $*$ Liberal is not significantly greater than zero for either analyst measure).

\section{Estimations Utilizing Country-Year Observations between 1992 and 2000}

To further rule out the possibility that our results simply capture trends in the data, we reestimate our primary tests restricting the analysis to the sample period 1992-2000. These estimations are a response to the possibility that I/B/E/S has increased its efforts to be a more comprehensive data source in recent time periods. We choose 1992 to start the restricted sample period, since

\footnotetext{
${ }^{18}$ Similar to our adjustments for the complete sample, we estimated the developed and emerging markets trend adjustment using both equal- and value-weighted (by coverage levels) averages. For parsimony, we only report results using equal-weighted averages. Results using value-weighted averages for our trend adjustment yield similar results. Finally, for our emerging market subsample, we only have one country (Brazil) that enforced insider trading restrictions prior to 1987. For robustness, we reestimated all tests after treating South Korea as a second control country for emerging markets (enforcement in 1988), and the results are quantitatively similar.
} 
in $1992 \mathrm{I} / \mathrm{B} / \mathrm{E} / \mathrm{S}$ significantly expanded both United States and international coverage. ${ }^{19}$ These estimations are presented in Table V.

Table V, Panel A documents that the levels results estimated for the period 1992-2000 for Exist and Enforce are qualitatively similar to the results documented in Table II, Panels A and B for the period 1987-2000. In particular, during this recent time period, both analyst measures increase upon enforcement of insider trading restrictions for emerging market countries, and the impact of enforcement is lower in countries that have liberalized relative to those that have not. Table V, Panel B reports a change specification for the period 1992-2000. Although these change results are weaker, they still generally support our hypothesis. In the estimations without interactions, the coefficient on $\Delta E$ Enforce is positive and significant for $\Delta$ Analyst, but not for $\Delta$ Proport. In the estimations with our Emerge interactions, the enforcement effect is positive and significant in emerging markets for $\Delta$ Proport, but not for $\Delta$ Analyst. Finally, for both analyst variables, the coefficient on enforcement is significantly greater than zero in countries that have not liberalized, and significantly lower in countries that have liberalized relative to those that have not.

\section{E. Investor Protection Levels and the Impact of Insider Trading Enforcement on Analysts}

In our final empirical analysis, we investigate whether the change in analyst following is larger or smaller when a country already has strong investor protections. We use a country's legal origin to proxy for the level of investor protection in a country (e.g., La Porta et al. (1998)). We classify countries according to whether their legal origin is primarily common law (English origin), civil law (French, German, or Scandinavian origin), or socialist (Soviet Union origin), by including an indicator for civil law (Civillaw) and socialist origin (Socialist) (data source: La Porta et al. (1999)). La Porta et al. conjecture that countries with a socialist origin have the most interventionist governments, next come countries with civil laws, and finally common law countries. We posit the same ordering for investor protection, with common law countries posited to have the best investor protections (La Porta et al. (1998)).

Descriptive statistics for our two legal origin variables are presented in Table I. Table VI presents coefficients from our baseline estimations after including these legal origin variables. Table VI documents that the enactment of insider laws in socialist countries is associated with a significant increase in analyst following. Moreover, the estimations show that our main results are robust to inclusion of legal origin. Without interactions, the coefficient on Enforce is positive and significant for both analyst measures. From the interaction analysis, we see that the increase in analyst following (both Analyst and Proport) upon insider trading enforcement is significantly higher in both civil law and socialist origin countries relative to common law countries. These results are consistent with diminishing returns to investor protections. That is, the enforcement of

\footnotetext{
${ }^{19}$ We thank an anonymous referee for identifying this event.
} 


\section{Table V}

Relation between Insider Trading Laws and Analyst Activity: Estimations Using Country-Year Observations between 1992 and 2000

This table presents select coefficients from various estimations of equations (1) and (2) (Panel A) and equation (3) (Panel B). The models in Panel A are estimated on a panel of 89 countries with a maximum of nine time-series observations (calendar years 1992 through 2000). The models in Panel B are estimated on a panel of 89 countries with a minimum (maximum) of 2 (8) time-series observations (i.e., annual changes between calendar years 1992 through 2000). All models are estimated using a pooled GLS estimation technique with annual indicator variables $\left(Y_{t}\right)$. All models are estimated using trend-adjusted analyst coverage data. Two-tailed $p$-values are presented in parentheses. All variables are defined in Appendix A. The superscripts "a" and "b" denote that the sum of coefficients on Exist and Enforce for emerging market countries (i.e., $\beta_{5}+\beta_{7}$ and $\beta_{6}+\beta_{8}$ ) are significantly different than zero at the 1 and $5 \%$ level, respectively, using a two-tailed $t$-test. The superscripts "c" and "d" denote that the sum of coefficients on Exist and Enforce for liberalized market countries (i.e., $\beta_{6}+\beta_{8}$ and $\beta_{7}+\beta_{9}$ ) are significantly different than zero at the 1 and $5 \%$ level, respectively, using a two-tailed $t$-test.

Panel A: Baseline Levels Regressions $(n=734)$

\begin{tabular}{|c|c|c|c|c|c|c|c|c|}
\hline \multirow{4}{*}{$\begin{array}{l}\text { Dependent } \\
\text { Variable }\end{array}$} & \multicolumn{4}{|c|}{$\log \left(\right.$ Analyst $\left._{i, t}\right)$} & \multicolumn{4}{|c|}{$\log \left(\right.$ Proport $\left._{i, t}\right)$} \\
\hline & \multicolumn{2}{|c|}{ Equation (1) } & \multicolumn{2}{|c|}{ Equation (2) } & \multicolumn{2}{|c|}{ Equation (1) } & \multicolumn{2}{|c|}{ Equation (2) } \\
\hline & 0.092 & -0.047 & 0.094 & 0.017 & 0.020 & -0.107 & 0.022 & 0.018 \\
\hline & $(0.065)$ & $(0.760)$ & $(0.062)$ & $(0.755)$ & $(0.100)$ & $(0.005)$ & $(0.078)$ & $(0.172)$ \\
\hline \multirow[t]{2}{*}{ Enforce } & 0.212 & -0.051 & 0.213 & 0.607 & 0.015 & -0.021 & 0.016 & 0.129 \\
\hline & $(0.000)$ & $(0.460)$ & $(0.000)$ & $(0.000)$ & $(0.187)$ & $(0.220)$ & $(0.147)$ & $(0.000)$ \\
\hline \multirow[t]{2}{*}{ Exist $*$ Emerge } & - & $0.150^{\mathrm{b}}$ & - & - & - & $0.137^{\mathrm{b}}$ & - & - \\
\hline & - & $(0.350)$ & - & - & - & $(0.001)$ & - & - \\
\hline \multirow[t]{2}{*}{ Enforce $*$ Emerge } & - & $0.429^{\mathrm{a}}$ & - & - & - & $0.060^{\mathrm{a}}$ & - & - \\
\hline & - & $(0.000)$ & - & - & - & $(0.003)$ & - & - \\
\hline \multirow[t]{2}{*}{ Exist $*$ Liberal } & - & - & - & $0.255^{\mathrm{c}}$ & - & - & - & -0.022 \\
\hline & - & - & - & $(0.011)$ & - & - & - & $(0.358)$ \\
\hline \multirow[t]{2}{*}{ Enforce $*$ Liberal } & - & - & - & -0.558 & - & - & - & $-0.160^{\mathrm{d}}$ \\
\hline & - & - & - & $(0.000)$ & - & - & - & $(0.000)$ \\
\hline$R^{2}$ & 0.2680 & 0.2971 & 0.2674 & 0.3055 & 0.1025 & 0.1177 & 0.1116 & 0.1579 \\
\hline
\end{tabular}

Panel B: Impact in Year of Change $(n=641)$

\begin{tabular}{|c|c|c|c|c|c|c|}
\hline \multirow{3}{*}{$\begin{array}{l}\text { Dependent Variable } \\
\Delta \text { Exist }\end{array}$} & \multicolumn{3}{|c|}{$\Delta \log \left(\right.$ Analyst $\left._{i, t}\right)$} & \multicolumn{3}{|c|}{$\Delta \log \left(\right.$ Proport $\left._{i, t}\right)$} \\
\hline & 0.033 & 0.194 & 0.014 & -0.011 & -0.037 & -0.002 \\
\hline & $(0.504)$ & $(0.166)$ & $(0.796)$ & $(0.440)$ & $(0.363)$ & $(0.910)$ \\
\hline \multirow[t]{2}{*}{$\Delta$ Enforce } & 0.085 & 0.103 & 0.309 & 0.013 & -0.032 & 0.089 \\
\hline & $(0.088)$ & $(0.194)$ & $(0.001)$ & $(0.345)$ & $(0.151)$ & $(0.001)$ \\
\hline \multirow[t]{2}{*}{$\Delta$ Exist $*$ Emerge } & - & -0.184 & - & - & 0.029 & - \\
\hline & - & $(0.219)$ & - & - & $(0.494)$ & - \\
\hline \multirow[t]{2}{*}{$\Delta$ Enforce $*$ Emerge } & - & -0.027 & - & - & $0.075^{\mathrm{b}}$ & - \\
\hline & - & $(0.787)$ & - & - & $(0.009)$ & - \\
\hline \multirow[t]{2}{*}{$\Delta$ Exist $*$ Liberal } & - & - & 0.098 & - & - & -0.036 \\
\hline & - & - & $(0.414)$ & - & - & $(0.299)$ \\
\hline \multirow[t]{2}{*}{$\Delta$ Enforce $*$ Liberal } & - & - & -0.317 & - & - & -0.106 \\
\hline & - & - & $(0.003)$ & - & - & $(0.001)$ \\
\hline$R^{2}$ & 0.0419 & 0.0440 & 0.0564 & 0.0612 & 0.0711 & 0.0767 \\
\hline
\end{tabular}




\section{Table VI}

\section{Evidence on Diminishing Returns to Legal Institutions}

This table presents select coefficients from estimations of the following model:

$$
\begin{aligned}
\log \left(\text { Analyst }_{i, t}\right){\operatorname{or~} \log \left(\text { Proport }_{i, t}\right)=} & \alpha_{1}+\sum_{t=1988}^{2000} \gamma_{t} Y_{t}+\beta_{1} \log \left(\text { GNP }_{i, t}\right)+\beta_{2} \text { Growth }_{\left(\text {GNP }_{i, t}\right)} \\
& +\beta_{3} \log \left(\text { Listed }_{i, t}\right)+\beta_{4} \text { Emerge }_{i, t}+\beta_{5} \text { Liberal }_{i, t} \\
& +\beta_{6} \text { Civillaw }_{i, t}+\beta_{7} \text { Socialist }_{i, t}+\beta_{8} \text { Exist }_{i, t}+\beta_{9} \text { Enforce }_{i, t} \\
& +\beta_{10} \text { Exist }_{i, t} * \text { Civillaw }_{i, t}+\beta_{11} \text { Enforce }_{i, t} * \text { Civillaw }_{i, t} \\
& +\beta_{12} \text { Exist }_{i, t} * \text { Socialist }_{i, t}+\beta_{13} \text { Enforce }_{i, t} * \text { Socialist }_{i, t}+\varepsilon_{i, t} .
\end{aligned}
$$

where $Y_{t}$ is a year indicator variable. This model is estimated on a panel of 94 countries with a maximum of 14 time-series observations (calendar years 1987 through 2000) using a pooled GLS estimation technique with annual indicator variables. All models are estimated using trendadjusted analyst coverage data. Two-tailed $p$-values are presented in parentheses. All variables are defined in Appendix A. $n=1,107$.

\begin{tabular}{lcccc}
\hline Dependent Variable & \multicolumn{2}{c}{$\log \left(\right.$ Analyst $\left._{i, t}\right)$} & \multicolumn{2}{c}{$\log \left(\right.$ Proport $\left._{i, t}\right)$} \\
\hline Civillaw & -0.235 & -0.257 & -0.003 & -0.013 \\
& $(0.222)$ & $(0.213)$ & $(0.932)$ & $(0.778)$ \\
Socialist & 0.188 & -0.059 & 0.018 & -0.063 \\
& $(0.418)$ & $(0.816)$ & $(0.707)$ & $(0.279)$ \\
Exist & 0.009 & -0.002 & -0.012 & -0.023 \\
& $(0.801)$ & $(0.969)$ & $(0.238)$ & $(0.143)$ \\
Enforce & 0.333 & 0.009 & 0.044 & -0.052 \\
& $(0.000)$ & $(0.904)$ & $(0.000)$ & $(0.006)$ \\
Exist $*$ Civillaw & - & -0.067 & - & $-0.012^{\mathrm{a}}$ \\
& - & $(0.320)$ & - & $(0.515)$ \\
Enforce $*$ Civillaw & - & $0.386^{\mathrm{a}}$ & - & $0.101^{\mathrm{a}}$ \\
& - & $(0.000)$ & - & $(0.000)$ \\
Exist $*$ Socialist & - & $0.214^{\mathrm{b}}$ & - & $0.063^{\mathrm{c}}$ \\
Enforce $*$ Socialist & - & $(0.044)$ & - & $(0.023)$ \\
Adj. $R^{2}$ & - & $0.628^{\mathrm{a}}$ & - & $0.241^{\mathrm{a}}$ \\
& - & $0.000)$ & 0.1679 & $(0.000)$ \\
\end{tabular}

a,b,c The sum of coefficients on Exist and Enforce for civil law countries (i.e., $\beta_{8}+\beta_{10}$ and $\beta_{9}+\beta_{11}$ ) and socialist countries (i.e., $\beta_{8}+\beta_{12}$ and $\beta_{9}+\beta_{13}$ ) are significantly different than zero at the 1 , 5 , and $10 \%$ level, respectively, using a two-tailed $t$-test.

insider trading restrictions in common law countries, which presumably have relatively high levels of preexisting investor protections, is associated with a smaller increase in analyst following than in civil and socialist origin countries.

\section{Summary and Conclusion}

We exploit cross-country and intertemporal variation in analyst following for 100 countries for the years 1987-2000 to test the hypothesis that analyst 
following increases upon the restriction of insider trading. Our main analysis tests whether analyst following increases following adoption of insider trading legislation and/or the initial enforcement of these laws.

We present evidence that analyst following as reported by I/B/E/S International increases upon initial enforcement of insider trading restrictions. We find that this increase is concentrated in emerging market countries. We find only modest evidence of a reaction around enactment of laws. These results hold in both levels and change specifications and are robust to inclusion of an array of control variables and rigorous specification checks. We take special care to rule out the possibility that our results simply capture a general upward trend in actual analyst coverage or in reported analyst coverage by I/B/E/S International. In particular, we trend-adjust analyst coverage for each country year using countries that enforced insider trading prior to our sample period as baseline countries, utilize bootstrapping procedures to test the statistical significance of our primary results, and reestimated the main regressions using only more recent time periods (1992-2000).

While we robustly document an increase in I/B/E/S-reported analyst following after initial enforcement of insider trading laws, care must be taken in interpreting this result. In particular, increases in analyst following reported by $\mathrm{I} / \mathrm{B} / \mathrm{E} / \mathrm{S}$ can capture the initiation of new coverage or the addition of a new contributors to I/B/E/S. Although we cannot disentangle the extent to which our documented relations capture the initiation of new coverage or the decisions of analysts to become contributors to I/B/E/S post-enforcement, both decisions represent an escalation in equity market participation by analysts and is consistent with the predictions of extant theory (Fishman and Hagerty (1992), Khanna et al. (1994), Bhattacharya et al. (2000), Brudney (1979), Ausubel (1990), and Leland (1992)). The fact that enforcement of insider trading laws creates incentives for analysts to initiate coverage, or to increase their prominence or prestige in the market by becoming a contributor to $\mathrm{I} / \mathrm{B} / \mathrm{E} / \mathrm{S}$, or for $\mathrm{I} / \mathrm{B} / \mathrm{E} / \mathrm{S}$ to increase efforts to expand the number of contributors to the database, is interesting and consistent with the theory that analyst behavior is an important channel through which insider trading restrictions act to reduce the cost of capital as documented in Bhattacharya and Daouk (2002).

Although we treat the enactment and enforcement of insider trading restrictions as exogenous shocks, there may exist important correlated omitted variables that cause both insider trading restrictions and analyst following. We document that our results are robust to controlling for several important country characteristics. First, we control for the date of countries' liberalization of their equity market (if they did liberalize). Our results are robust to inclusion of the date of liberalization, and we find that the increase in analyst following after insider trading enforcement is smaller in countries that already have liberalized. Second, we also document that our results are also robust to the inclusion of two direct measures of country-level financial flows: foreign direct investment and trade flows.

Our results also add to the growing body of evidence that financial development and legal regimes are intricately linked (e.g., Beck et al. (2001, 2003), 
Demirgüç-Kunt and Maksimovic (1998), La Porta et al. (1997, 1998), and Lombardo and Pagano (1999)). We investigate whether analyst following increases more or less upon a shift in the insider trading regime in countries that already have strong investor protections, as measured by common law legal origin. We document that the increase in analyst following upon enforcement of insider trading restrictions is significantly higher in both civil law and socialist origin countries relative to common law countries. We also find a significant increase in analyst following upon enactment of insider trading laws in socialist countries. These results are consistent with diminishing returns to investor protections.

In conclusion, we believe that this cross-country, panel-based evidence of a positive relation between analyst following and insider trading restrictions is interesting, especially given the predictions of the crowding-out theory and recent empirical evidence by Bhattacharya and Daouk (2002) and by Bhattacharya et al. (2000). Although we cannot completely rule out alternative explanations to the crowding-out theory, our empirical design had the potential to cast meaningful doubt on the empirical validity of the underlying theory if no positive relation between analyst following and insider trading restrictions had been detected.

Appendix A

\begin{tabular}{|c|c|c|}
\hline Variable & Definition & Source \\
\hline Analyst & $\begin{array}{l}\text { Mean number of analysts providing a } \\
\text { forecast for each firm listed on I/B/E/S in } \\
\text { a given calendar year }\end{array}$ & I/B/E/S International \\
\hline Ibesfirm & $\begin{array}{l}\text { Number of firms in a country that are } \\
\text { included on I/B/E/S in a given calendar } \\
\text { year }\end{array}$ & I/B/E/S International \\
\hline Proport & $\begin{array}{l}\text { Proportion of firms in a country covered by } \\
\text { an analyst in a given calendar year } t . \\
\text { Defined as } \text { Ibesfirm }_{i, t} / \text { Listed }_{i, t}\end{array}$ & I/B/E/S International \\
\hline Listed & $\begin{array}{l}\text { Number of domestic companies in a country } \\
\text { listed on the home country's stock } \\
\text { exchange during a given calendar year }\end{array}$ & Datastream \\
\hline $\log (\cdot)$ & $\begin{array}{l}\text { The log of each analyst coverage variable is } \\
\text { calculated as the log of one plus the } \\
\text { respective raw realization of that } \\
\text { variable }\end{array}$ & \\
\hline Exist $_{i, t}$ & $\begin{array}{l}\text { An indicator variable equal to one if the } \\
\text { country enacted insider trading laws } \\
\text { prior to or during calendar year } t \text {, zero } \\
\text { otherwise }\end{array}$ & Bhattacharya and Daouk (2002) \\
\hline Enforce $_{i, t}$ & $\begin{array}{l}\text { An indicator variable equal to one if the } \\
\text { country enforced its first insider trading } \\
\text { case prior or during calendar year } t \text {, zero } \\
\text { otherwise }\end{array}$ & Bhattacharya and Daouk (2002) \\
\hline
\end{tabular}




\section{Appendix A-Continued}

\begin{tabular}{|c|c|c|}
\hline Variable & Definition & Source \\
\hline$\Delta$ Exist $_{i, t}$ & $\begin{array}{l}\text { An indicator variable equal to one if the } \\
\text { country enacted insider trading laws in } \\
\text { calendar year } t \text {, zero otherwise. }\end{array}$ & Bhattacharya and Daouk (2002) \\
\hline$\Delta$ Enforce $_{i, t}$ & $\begin{array}{l}\text { An indicator variable equal to one if the } \\
\text { country enforced it's first insider trading } \\
\text { law in calendar year } t \text {, zero otherwise. }\end{array}$ & Bhattacharya and Daouk (2002) \\
\hline$Y_{t}$ & $\begin{array}{l}\text { An indicator variable equal to one if the } \\
\text { country-year observation relates to } \\
\text { calendar year } t \text {, zero otherwise. }\end{array}$ & \\
\hline $\log \left(G N P_{i, t}\right)$ & $\begin{array}{l}\text { The natural logarithm of country } i \text { 's per } \\
\text { capita GNP for calendar year } t \text {. }\end{array}$ & World Development Indicators \\
\hline $\operatorname{Growth}\left(G N P_{i, t}\right)$ & $\begin{array}{l}\text { Annual growth rate in real GNP between } \\
\text { year } t-1 \text { and } t \text {. }\end{array}$ & World Development Indicators \\
\hline Emerge & $\begin{array}{l}\text { An indicator variable equal to one if the } \\
\text { country was not treated as a developed } \\
\text { country, zero otherwise. The } 22 \text { countries } \\
\text { that are classified as developed are: } \\
\text { Australia, Austria, Belgium, Canada, } \\
\text { Denmark, Finland, France, Germany, } \\
\text { Hong Kong, Ireland, Italy, Japan, } \\
\text { Luxembourg, Netherlands, Norway, New } \\
\text { Zealand, Singapore, Spain, Sweden, } \\
\text { Switzerland, Great Britain, and United } \\
\text { States. All remaining countries are } \\
\text { classified as emerging. }\end{array}$ & Bhattacharya and Daouk (2002) \\
\hline Liberal $_{i, t}$ & $\begin{array}{l}\text { An indicator variable equal to one if the } \\
\text { country's capital market liberalization } \\
\text { date preceded or is during calendar year } \\
t, \text { zero otherwise. }\end{array}$ & Bekaert et al. (2001) \\
\hline Civillaw & $\begin{array}{l}\text { An indicator variable equal to one if the } \\
\text { country's legal origin is French, German, } \\
\text { or Scandinavian, zero otherwise. }\end{array}$ & La Porta et al. (1999) \\
\hline Socialist & $\begin{array}{l}\text { An indicator variable equal to one if the } \\
\text { country's legal origin is socialist in } \\
\text { nature, zero otherwise. }\end{array}$ & La Porta et al. (1999) \\
\hline$F D I_{i, t}$ & $\begin{array}{c}\text { The amount of foreign direct investment in } \\
\text { country } i \text { during calendar year } t \text {, scaled } \\
\text { by the country's gross domestic product. }\end{array}$ & World Development Indicators \\
\hline Open $_{i, t}$ & $\begin{array}{l}\text { Trade openness, measured as the sum of } \\
\text { total imports and exports for country } i \text { in } \\
\text { calendar year } t \text {, scaled by the country's } \\
\text { gross domestic product. }\end{array}$ & World Development Indicators \\
\hline
\end{tabular}




\section{Appendix B. Dates of Establishment of Main Exchange, Enactment, and Enforcement of Insider Trading Laws and Liberalization of the Markets}

\begin{tabular}{|c|c|c|c|c|c|}
\hline Country & $\begin{array}{l}\text { Country } \\
\text { Code }\end{array}$ & $\begin{array}{c}\text { Establishment } \\
\text { of Main } \\
\text { Exchange }\end{array}$ & $\begin{array}{l}\text { IT Laws } \\
\text { Existence }\end{array}$ & $\begin{array}{c}\text { IT Laws } \\
\text { Enforcement }\end{array}$ & $\begin{array}{c}\text { Liberalization } \\
\text { Date }\end{array}$ \\
\hline \multicolumn{6}{|c|}{ Developed Countries } \\
\hline Australia & AUS & 1859 & 1991 & 1996 & Before 1969 \\
\hline Austria & AUT & 1771 & 1993 & - & Before 1969 \\
\hline Belgium & BEL & 1801 & 1990 & 1994 & Before 1969 \\
\hline Canada & CAN & 1878 & 1966 & 1976 & Before 1969 \\
\hline Denmark & DNK & 1919 & 1991 & 1996 & Before 1969 \\
\hline Finland & FIN & 1912 & 1989 & 1993 & Before 1969 \\
\hline France & FRA & 1826 & 1967 & 1975 & Before 1969 \\
\hline Germany & $\mathrm{DEU}$ & 1585 & 1994 & 1995 & Before 1969 \\
\hline Hong Kong & HKG & 1891 & 1991 & 1994 & Before 1969 \\
\hline Ireland & IRL & 1973 & 1990 & - & Before 1969 \\
\hline Italy & ITA & 1808 & 1991 & 1996 & Before 1969 \\
\hline Japan & JPN & 1878 & 1988 & 1990 & 1980 \\
\hline Luxembourg & LUX & 1929 & 1991 & - & Before 1969 \\
\hline Netherlands & NLD & 1600 & 1989 & 1994 & Before 1969 \\
\hline New Zealand & NZL & 1870 & 1988 & - & 1984 \\
\hline Norway & NOR & 1819 & 1985 & 1990 & Before 1969 \\
\hline Singapore & SGP & 1930 & 1973 & 1978 & Before 1969 \\
\hline Spain & ESP & 1831 & 1994 & 1998 & 1978 \\
\hline Sweden & SWE & 1863 & 1971 & 1990 & Before 1969 \\
\hline Switzerland & $\mathrm{CHE}$ & 1938 & 1988 & 1995 & Before 1969 \\
\hline United Kingdom & GBR & 1773 & 1980 & 1981 & Before 1969 \\
\hline United States & USA & 1792 & 1934 & 1961 & Before 1969 \\
\hline \multicolumn{6}{|c|}{ Emerging Markets } \\
\hline Argentina & ARG & 1854 & 1991 & 1995 & 1989 \\
\hline Armenia & $\mathrm{ARM}$ & 1993 & 1993 & - & - \\
\hline Bahrain & BHR & 1987 & 1990 & - & - \\
\hline Bangladesh & BGD & 1954 & 1995 & 1998 & - \\
\hline Barbados & $\mathrm{BRB}$ & 1987 & 1987 & - & - \\
\hline Bermuda & $\mathrm{BMU}$ & 1971 & - & - & - \\
\hline Bolivia & BOL & 1979 & - & - & - \\
\hline Botswana & BWA & 1989 & - & - & - \\
\hline Brazil & BRA & 1890 & 1976 & 1978 & 1991 \\
\hline Bulgaria & BGR & 1991 & - & - & - \\
\hline Chile & CHL & 1893 & 1981 & 1996 & 1992 \\
\hline China & $\mathrm{CHN}$ & 1990 & 1993 & - & - \\
\hline Colombia & $\mathrm{COL}$ & 1928 & 1990 & - & 1991 \\
\hline Costa Rica & CRI & 1976 & 1990 & - & - \\
\hline Croatia & HRV & 1918 & 1995 & - & - \\
\hline Cyprus & CYP & 1996 & 1999 & - & - \\
\hline Czech Republic & $\mathrm{CZE}$ & 1871 & 1992 & 1993 & - \\
\hline Ecuador & ECU & 1969 & 1993 & - & - \\
\hline Egypt & EGY & 1890 & 1992 & - & - \\
\hline El Salvador & SLV & 1992 & - & - & - \\
\hline Estonia & EST & 1996 & 1996 & - & - \\
\hline Ghana & GHA & 1989 & 1993 & - & - \\
\hline Greece & GRC & 1876 & 1988 & 1996 & 1987 \\
\hline Guatemala & GTM & 1986 & 1996 & - & - \\
\hline Honduras & HND & 1992 & 1988 & - & - \\
\hline
\end{tabular}


Appendix B-Continued

\begin{tabular}{|c|c|c|c|c|c|}
\hline Country & $\begin{array}{l}\text { Country } \\
\text { Code }\end{array}$ & $\begin{array}{c}\text { Establishment } \\
\text { of Main } \\
\text { Exchange }\end{array}$ & $\begin{array}{l}\text { IT Laws } \\
\text { Existence }\end{array}$ & $\begin{array}{c}\text { IT Laws } \\
\text { Enforcement }\end{array}$ & $\begin{array}{c}\text { Liberalization } \\
\text { Date }\end{array}$ \\
\hline Hungary & HUN & 1864 & 1994 & 1995 & - \\
\hline Iceland & ISL & 1985 & 1989 & - & - \\
\hline India & IND & 1875 & 1992 & 1998 & 1992 \\
\hline Indonesia & IDN & 1912 & 1991 & 1996 & 1989 \\
\hline Iran & IRN & 1966 & - & - & - \\
\hline Israel & ISR & 1953 & 1981 & 1989 & - \\
\hline Jamaica & JAM & 1961 & 1993 & - & - \\
\hline Jordan & JOR & 1978 & - & - & 1995 \\
\hline Kazakhstan & $\mathrm{KAZ}$ & 1997 & 1996 & - & - \\
\hline Kenya & $\mathrm{KEN}$ & 1954 & 1989 & - & - \\
\hline Kuwait & KWT & 1984 & - & - & - \\
\hline Latvia & LVA & 1993 & - & - & - \\
\hline Lebanon & $\mathrm{LBN}$ & 1920 & 1995 & - & - \\
\hline Lithuania & LTU & 1926 & 1996 & - & - \\
\hline Macedonia & MKD & 1996 & 1997 & - & - \\
\hline Malawi & MWI & 1996 & - & - & - \\
\hline Malaysia & MYS & 1973 & 1973 & 1996 & 1988 \\
\hline Malta & MLT & 1992 & 1990 & - & - \\
\hline Mauritius & MUS & 1988 & 1988 & - & - \\
\hline Mexico & MEX & 1894 & 1975 & - & 1989 \\
\hline Moldova & MDA & 1994 & 1995 & - & - \\
\hline Mongolia & MNG & 1991 & 1994 & - & - \\
\hline Morocco & MAR & 1929 & 1993 & - & - \\
\hline Namibia & NAM & 1992 & - & - & - \\
\hline Nigeria & NGA & 1960 & 1979 & - & 1995 \\
\hline Oman & $\mathrm{OMN}$ & 1988 & 1989 & 1999 & - \\
\hline Pakistan & PAK & 1947 & 1995 & - & 1991 \\
\hline Panama & PAN & 1990 & 1996 & - & - \\
\hline Paraguay & PRY & 1977 & 1999 & - & - \\
\hline Peru & PER & 1951 & 1991 & 1994 & - \\
\hline Philippines & PHL & 1927 & 1982 & - & 1991 \\
\hline Poland & POL & 1817 & 1991 & 1993 & - \\
\hline Portugal & PRT & 1825 & 1986 & - & 1986 \\
\hline Romania & $\mathrm{ROM}$ & 1882 & 1995 & - & - \\
\hline Russia & RUS & 1994 & 1996 & - & - \\
\hline Saudi Arabia & SAU & 1984 & 1990 & - & - \\
\hline Slovakia & SVK & 1991 & 1992 & - & - \\
\hline Slovenia & SVN & 1924 & 1994 & 1998 & - \\
\hline South Africa & $\mathrm{ZAF}$ & 1887 & 1989 & - & - \\
\hline South Korea & KOR & 1956 & 1976 & 1988 & 1992 \\
\hline Sri Lanka & LKA & 1896 & 1987 & 1996 & - \\
\hline Swaziland & SWZ & 1990 & - & - & - \\
\hline Tanzania & TZA & 1998 & 1994 & - & - \\
\hline Thailand & THA & 1974 & 1984 & 1993 & 1987 \\
\hline Trinidad and Tobago & TTO & 1981 & 1981 & - & - \\
\hline Tunisia & TUN & 1969 & 1994 & - & - \\
\hline Turkey & TUR & 1866 & 1981 & 1996 & 1989 \\
\hline Ukraine & UKR & 1992 & - & - & - \\
\hline Uruguay & URY & 1867 & 1996 & - & - \\
\hline Uzbekistan & UZB & 1994 & - & - & - \\
\hline Venezuela & VEN & 1840 & 1998 & - & 1990 \\
\hline Zambia & ZMB & 1994 & 1993 & - & - \\
\hline Zimbabwe & ZWE & 1896 & - & - & 1993 \\
\hline
\end{tabular}




\section{REFERENCES}

Ausubel, Lawrence, 1990, Insider trading in a rational expectations economy, American Economic Review 80, 1022-1041.

Ayers, Benjamin, and Robert Freeman, 2003, Evidence that analyst following and institutions accelerate the pricing of future earnings, Review of Accounting Studies 8, 47-68.

Barry, Christopher B., and Stephen J. Brown, 1985, Differential information and security market equilibrium, Journal of Financial and Quantitative Analysis 20, 407-422.

Beck, Thorsten, Asli Demirgüç-Kunt, and Ross Levine, 2001, Law, politics, and finance, Working paper, World Bank.

Beck, Thorsten, Asli Demirgüç-Kunt, and Ross Levine, 2003, Law, endowments, and finance, Journal of Financial Economics 70, 137-181.

Bekaert, Geert, Campbell Harvey, and Christian Lundblad, 2001, Emerging equity markets and economic growth, Journal of Development Economics 66, 465-504.

Beny, Laura, 1999, A comparative empirical investigation of agency and market theories of insider trading, John M. Olin Center for Law, Economics and Business Discussion paper 264, Harvard University.

Bhattacharya, Utpal, and Hazem Daouk, 2002, The world price of insider trading, Journal of Finance 57, 75-108.

Bhattacharya, Utpal, Hazem Daouk, Brian Jorgenson, and Carl-Heinrich Kehr, 2000, When an event is not an event: The curious case of an emerging market, Journal of Financial Economics $55,69-101$.

Brennan, Michael, Narasimhan Jegadeesh, and Bhaskaran Swaminathan, 1993, Investment analysis and the adjustment of stock prices to common information, Review of Financial Studies 6 , 799-824.

Brennan, Michael, and Avanidhar Subrahmanyam, 1995, Investment analysis and the price formation in securities markets, Journal of Financial Economics 38, 361-381.

Bris, Artura, 2000, Do insider trading laws work? ICF Working paper No. 00-19, Yale University.

Brudney, Victor, 1979, Insiders, outsiders, and informational advantages under the federal securities laws, Harvard Law Review 93, 322.

Bushman, Robert, Joseph Piotroski, and Abbie Smith, 2004, What determines corporate transparency? Journal of Accounting Research 42, 207-252.

DeMarzo, Peter M., Michael Fishman, and Kathleen Hagerty, 1998, The optimal enforcement of insider trading regulations, Journal of Political Economy 106, 602-632.

Demirgüç-Kunt, Asli, and Vojislav Maksimovic, 1998, Law, finance and firm growth, Journal of Finance 53, 2107-2137.

Easley, David, Soeren Hvidkjaer, and Maureen O'Hara, 2002, Is information risk a determinant of asset returns? Journal of Finance 57, 2185-2221.

Easley, David, and Maureen O'Hara, 2004, Information and the cost of capital, Journal of Finance $59,1553-1583$.

Easley, David, Maureen O’Hara, and Joseph Paperman, 1998, Financial analysts and informationbased trade, Journal of Financial Markets 1, 175-201.

Estrada, Javier, and J. Ignacio Pena, 2001, Empirical evidence on the impact of European insider trading regulations, Studies in Economics and Finance 20, 12-34.

Fishman, Michael, and Kathleen Hagerty, 1992, Insider trading and the efficiency of stock prices, Rand Journal of Economics 23, 106-122.

Hong, Harrison, Terence Lim, and Jeremy Stein, 2000, Bad news travels slowly: Size, analyst coverage and the profitability of momentum strategies, Journal of Finance 55, 265-296.

Keown, Arthur, and John Pinkerton, 1981, Merger announcements and insider trading activity: An empirical investigation, Journal of Finance 36, 855-869.

Khanna, Naveen, Steve Slezak, and Michael Bradley, 1994, Insider trading, outside search, and resource allocation: Why firms and society may disagree on insider trading restrictions, Review of Financial Studies 7, 163-193.

Kyle, Albert, 1985, Continuous auctions and insider trading, Econometrica 53, 1315-1335. 
La Porta, Rafael, Florencio Lopez-de-Silanes, Andrei Shleifer, and Robert Vishny, 1997, Legal determinants of external capital, Journal of Finance 52, 1131-1150.

La Porta, Rafael, Florencio Lopez-de-Silanes, Andrei Shleifer, and Robert Vishny, 1998, Law and finance, Journal of Political Economy 106, 1113-1155.

La Porta, Rafael, Florencio Lopez-de-Silanes, Andrei Shleifer, and Robert Vishny, 1999, The quality of government, Journal of Law, Economics and Organization 15, 222-279.

Laeven, Luc, and Enrico Perotti, 2001, Confidence building in emerging markets, CEPR Discussion paper no. 3055.

Leland, Hayne E., 1992, Insider trading: Should it be prohibited? Journal of Political Economy 100, $859-887$.

Lobo, Gerald, and Amal A. W. Mahmoud, 1989, Relationship between differential amounts of prior information and security return variability, Journal of Accounting Research 27, 116-134.

Lombardo, Davide, and Pagano Marco, 1999, Legal determinants of the return on equity, CEPR Discussion paper no. 2276 and CSEF Working paper no. 24, Università di Salerno.

Maug, Ernst, 1999, Insider trading legislation and corporate governance, Working paper, Duke University.

Merton, Robert C., 1987, A simple model of capital market equilibrium with incomplete information, Journal of Finance 42, 483-510.

Meulbroek, Lisa K., 1992, An empirical analysis of illegal insider trading, Journal of Finance 47, 1661-1699.

Piotroski, Joseph, 2000, Value investing: The use of historical financial statement information to separate winners from losers, Journal of Accounting Research 38, 1-41

Piotroski, Joseph, and Darren Roulstone, 2004, The influence of analysts, institutional investors and insiders on the incorporation of market, industry and firm-specific information into stock prices, The Accounting Review 79, 1119-1151.

Rajan, Raghuram, and Luigi Zingales, 2002, The great reversals: The politics of financial development in the twentieth century, Journal of Financial Economics 69, 5-50.

Roulstone, Darren, 2003, Analyst following and market liquidity, Contemporary Accounting Research 20, 1-27.

Shin, Jhinyoung, 1996, The optimal regulation of insider trading, Journal of Financial Intermediation 5, 49-73. 